\title{
Interactions and Pressure Functionals for Disordered Lattice Systems
}

\author{
Boguslav Zegarlinski * \\ Mathematics Department, Ruhr-Universität Bochum, W-4630 Bochum 1, \\ Federal Republic of Germany
}

Received November 1, 1989; in revised form March 1, 1991

\begin{abstract}
The purpose of this paper is to provide a theoretical framework for disordered spin systems on a lattice, similar to that of classical statistical mechanics in the sense of Ruelle [Ru]. We prove the existence of a continuous pressure functional on a large Banach space of random interactions (highly generalizing the classical one) and formulate an analog of the variational principle.
\end{abstract}

\section{Introduction}

The statistical mechanics of disordered lattice systems, considered as a mathematical description of models of real materials such as e.g. real crystals, alloys of magnetic and nonmagnetic metals, glasses and others, have to deal with new phenomena and problems. For general reviews and connection to other domains of physics, mathematics and biology see e.g. [4, 25, 36, 48, 11$]$.

It was observed by Edwards and Anderson [15], that it is very useful to describe such systems as a lattice model with random interactions. The randomness and existence of competing interactions allow a system to behave thermodynamically well even if the decay of interactions is very slow and its amplitude is a'priori unbounded. The first rigorous proof of this fact has been given in $[40,33]$, where the existence of a (nonrandom) thermodynamic limit of the pressure has been proven for a random bond Ising model given by a hamiltonian function

$$
H_{0} \equiv-\sum_{i \neq j} J_{i j} \sigma_{i} \sigma_{j} ; \quad i, j \in \mathbb{Z}^{d}
$$

with $\left\{J_{i j}\right\}$ being independent random variables satisfying for all $i, j \in \mathbb{Z}^{d}, i \neq j$,

and for all $n \in \mathbb{N}$

$$
E J_{i j}=0 \text {, }
$$

$$
\left|E J_{i j}^{n}\right| \leqq n ! \gamma^{n}|i-j|^{-n \alpha d}
$$

with some constants $0<\gamma<\infty$ and $\frac{1}{2}<\alpha<\infty$.

\footnotetext{
* Supported by SFB 237
} 
Note that for $\frac{1}{2}<\alpha \leqq 1$ we can have (non-classical) long range interactions satisfying

$$
\sum_{j}\left|J_{i j}\right|=\infty
$$

with probability one. Afterwards a simpler proof of that, based on use of subadditive ergodic theorem, appeared in [20]. The generalization to the case when $(0.2 b)$ is satisfied only up to $n=4$ has been given in [58]. It was observed in [17] that in the special case when the free measure is symmetric one may apply the subadditive ergodic theorem and show that actually the estimates of [58] are sufficient to include the case when $(0.2 b)$ holds only for $n \leqq 2$.

The other example of a long range interaction (in the sense that an analog of $(0.3)$ is satisfied) is provided by the Sherrington-Kirkpatrick model [55]. In this case, at high temperatures, the infinite volume pressure has been exactly computed in $[1,31]$.

(For interactions with $\alpha>1$ one can find related results in $[23,57,44]$; see also [12 and 49].)

Originally the consideration of random interactions satisfying (0.1)- $(0.3)$ was motivated by RKKY interactions given by

$$
J_{i j} \equiv G_{i j} n_{i} n_{j}, \quad i, j \in \mathbb{Z}^{d}
$$

with $\left\{n_{i}\right\}$ independent occupation number random variables and $G_{i j}$ defined in $d=3$ dimensions for $i \neq j$ by

$$
G_{i j}=\frac{-q_{F}|i-j| \cos \left(q_{F}|i-j|\right)+\sin \left(q_{F}|i-j|\right)}{|i-j|^{4}} .
$$

The fact that $G_{i j}$ changes its sign was modeled by $(0.2 \mathrm{a})$. Obviously $(0.3)$ is satisfied for any translation invariant distribution of occupation variables not concentrated on the empty configuration. The original RKKY interaction is not thermodynamically stable; however, as was proven in [32], there is a class of reasonable interactions sharing its essential features. This class is contained in the set of interactions given by $(0.4)$ with

$$
G_{i j} \equiv \frac{1}{(2 \pi)^{d}} \int_{(-\pi, \pi)^{d}} d q e^{i q(i-j)} \hat{G}(q)
$$

where

$$
0 \leqq \hat{G}(q) \leqq\|\widehat{G}\|_{\infty}<\infty .
$$

In the present paper we generalise the above mentioned results concerning the pressure, showing that thermodynamics is well defined, in the sense of existence of a nonrandom infinite volume pressure functional, for a much larger class of interactions.

A description of the simplest disordered systems with a Hamiltonian of the type

$$
H \equiv H_{0}+\lambda \sum_{i} h_{i} \sigma_{i}
$$

(where the magnetic field variables $\left\{h_{i}\right\}_{i \in \mathbb{Z}^{d}}$ are independent and identically distributed, and $0 \leqq \lambda<\infty)$ is not an easy task even for short range interactions. (By a short range interaction we mean here the interaction for which the quantity on the left-hand side of $(0.3)$ is finite with probability one.)

There are several interesting results concerning this case: The proofs of absence of phase transition for one dimensional Ising models of type $(0.7)$ with $1<\alpha$ have 
been given in $[21,39,9,8,7]$. This is in contradistinction to nonrandom classical models where e.g. for ferromagnetic models the phase transition occur for $1<\alpha \leqq 2$, see $[14,27]$.

The $O(N)$ random models have been investigated in the series of papers [52, 53, $18,16,5]$, successively extending the region of nonbroken $O(N)$ symmetry up to interactions with $\alpha=1$ for $d=1,2$. Note that for the nonrandom case with ferromagnetic couplings in two dimensions one has symmetry breaking for $1<\alpha<2$ [43] and symmetry remains unbroken for $\alpha \geqq 2$, whereas for $d=3$ one has symmetry breaking even for finite range interactions [28].

For an Ising model with nearest neighbours random interaction at high temperature or large magnetic field, the cluster property and uniqueness result has been obtained in [3] by the use of a cluster expansion method. A very general and strong method for studying the Ising models with short range interactions has been developed in [26]. The authors consider the following important cases: a) high temperature spin glasses, b) low temperature nearest neighbours random field Ising models (RFIM's) with sufficiently large covariance of the random magnetic field (large disorder), c) low temperature predominantly ferromagnetic spin glasses. For all these cases it is shown that the truncated correlation functions have exponential tree decay with probability one. By this, for a) and b), they prove the existence of a disordered phase and (strong) uniqueness of the corresponding Gibbs measures, in regions full of Griffiths singularities [34] where the conventional high temperature cluster expansion diverges.

The hardest problem was the RFIM's with small covariance of the random magnetic field at low temperatures. This was resolved for $d=3$ dimensions in [6], (after preparatory important works $[24,37,10]$ ), where the existence of ferromagnetic phases in the low temperature region is shown. As argued in [24] for $d \leqq 2$, the random magnetic field even with small covariance should cause disorder at any temperature. Recently this has been rigorously proven in [2].

The Ising models with long range interactions [in the sense of $(0.3)]$ of random bound type have been considered in $[29,30]$ where existence of a disordered phase, weak uniqueness and clustering were proven and in [58] where a kind of strong uniqueness has been shown. (For related results for the Sherrington-Kirkpatrick model, see $[1,31]$.) The random site Ising model with long range interactions, defined by $(0.1)$ and $(0.6)$, has been discussed in [32]. It was shown there that if the temperature $\beta^{-1}$ satisfies

$$
\beta\|\widehat{G}\|_{\infty}<1,
$$

then the corresponding spin system is in the disordered phase (in the sense that the Edwards-Anderson parameter equals zero), where it has a decay of correlations similar to that of the interaction. Moreover the infinite volume limit of physical observables is independent of boundary conditions.

Although the description of the high temperature region for long range interactions could seem to be quite complete, there are still a lot of interesting questions concerning the disordered phase to answer. (For example the $C^{\infty}$ property of pressure implied by sufficiently strong tree decay of truncated correlation functions. The other problem concerns the behaviour at any temperature but in a large magnetic field.)

The low temperature region for long range interactions is terra incognita (but see [1]). To study this region and phase transition phenomena it is useful to have an a priori definition of an infinite volume state of spin system. For short range 
interactions this is provided by the definition of Gibbs measure $[13,45,22,53]$ and concerning disordered systems requires no additional modification. For the case of long range interactions it is not possible to use the Gibbsian description, since we have no well defined local specification on the space of all spin configurations (for some discussions see e.g. [58]). In the present paper we show that it is possible to define an infinite volume equilibrium state for a random system by use of a variational principle. Note that contrary to the non-random case $[54,38]$ it is necessary to use it even for some interactions of finite body type (e.g. two point interactions). We develop in a sense a fully fledged approach based on a continuous pressure functional defined on a Banach space of random interactions. Therefore one can hope to extend the theory of phase transitions given in [38] to our case. This would be important, since up to now, the existence of generic spin glass phase transitions remains unproven. (However there is evidence for that in the mean field model of Sherrington-Kirkpatrick given in [1].)

Let us remark that as a matter of fact we have quite a poor understanding of phase transitions for long range (in the classical sense) interactions besides the ferromagnetic one. As a first step in the direction of the constructive description of spin systems with long range interactions one can view the work [50], in which a class of polynomially decaying interactions with a "small" long range part is considered.

The organization of our paper is as follows. In the first section we introduce notation and define the Banach spaces of interactions we want to consider. The second section is devoted to the proof of existence of nonrandom continuous pressure functionals defined on Banach space of "random bond" type interactions. In Sect. 3 we get similar results for replica systems. Section 4 is used to formulate a corresponding variational principle. In the last section we discuss the possible reasonable extensions of long range "random site" type interactions (0.4) and (0.6) and prove for some class of them the existence and continuity of the infinite volume pressure for spin systems (including replica systems).

\section{Definitions and Notations}

Let $\Gamma$ be a countable set called a lattice. It is assumed that there is an infinite abelian group of bijections of $\boldsymbol{\Gamma}$, denoted by $\mathbf{T}$ and called a translation group. Let $\left\{e_{l} \in \mathbf{T}: l=1, \ldots, d\right\}, d \in \mathbb{N}$, be a set of generators of $\mathbf{T}$, i.e. each element $a \in \mathbf{T}$ can be written as

$$
a \equiv \sum_{l=1}^{d} n_{l} e_{l}
$$

with some $n_{l} \in \mathbb{Z}, l=1, \ldots, d$. A translation of $i \in \boldsymbol{\Gamma}$ by an element $a \in \mathbf{T}$ is denoted by $i+a$. A translation $T_{a} \Lambda$ of a set $\Lambda \subset \Gamma$ is by definition the set

$$
T_{a} \Lambda \equiv\{i \in \Gamma: i-a \in \Lambda\}
$$

In general the translation group $\mathbf{T}$ need not act transitively on $\boldsymbol{\Gamma}$. We assume only that there is a smallest finite set $\Delta C \Gamma$, called an elementary cell of $\Gamma$, such that for any $a, a^{\prime} \in \mathbf{T}$

$$
a \neq a^{\prime} \Rightarrow T_{a} \Delta \cap T_{a^{\prime}} \Delta=\emptyset
$$


and

$$
\Gamma=\bigcup_{a \in \mathbf{T}} T_{a} \Delta
$$

Note that the choice of $\Delta$ can be in general nonunique.

Let $\mathscr{F}$ be the family of finite sets in $\Gamma$. By $|\Lambda|$ we denote the number of elements in $\Lambda \in \mathscr{F}$ and by $\bar{\Lambda}$ a smallest covering of $\Lambda \in \mathscr{F}$ by elementary cells. For $i \in \Gamma$ and a set $\Lambda \subset \boldsymbol{\Gamma}$ we define a distance of $i \in \boldsymbol{\Gamma}$ from $\Lambda$ by

$$
d(i, \Lambda) \equiv \inf \left\{\sum_{l=1}^{d}\left|n_{l}\right|: T_{a} i \in \bar{\Lambda}, a \equiv \sum_{l=1}^{d} n_{l} e_{l}\right\} .
$$

The diameter of $\Lambda \in \mathscr{F}$ by definition equals

$$
\operatorname{diam}(\Lambda)=\sup _{i, j \in \Lambda}(d(i, j)+1)
$$

An increasing and absorbing sequence $\mathscr{F}_{0} \equiv\left\{\Lambda_{n} \in \mathscr{F}\right\}_{n \in \mathbb{N}}$ is called a countable base, i.e. we have $\Lambda_{n} \subset \Lambda_{n+1}$ for any $n \in \mathbb{N}$ and for any $\Lambda \in \mathscr{F}$ there is $n_{0} \in \mathbb{N}$ such that $\Lambda \subset \Lambda_{n}$ for any $n \geqq n_{0}$.

Further we will use special countable bases called van Hove sequences. To define them, let us first introduce for any $N \in \mathbb{N}, N>1$ the sets

$$
\Delta_{N} \equiv\left\{\bigcup T_{a} \Delta: a=\sum_{l=1}^{d} n_{l} e_{l},\left|n_{l}\right| \leqq N\right\} .
$$

The boundary $\partial \Delta_{N}$ of $\Delta_{N}$ is defined to be

$$
\partial \Delta_{N} \equiv\left\{i \in \Delta_{N}: \exists 1 \leqq l \leqq d,\left|n_{l}\right|=1 \text { that } i+n_{l} e_{l} \notin \Delta_{N}\right\} .
$$

Similarly one can define $\partial \Lambda$ for any $\Lambda \in \mathscr{F}$.

From the definitions (1.5) and (1.6) we have

$$
\lim _{N \rightarrow \infty} \frac{\left|\partial \Delta_{N}\right|}{\left|\Delta_{N}\right|}=0
$$

For $\Lambda \in \mathscr{F}$ and $N \in \mathbb{N}$ we set

$$
\Lambda_{N}^{+} \equiv\left\{\bigcup T_{a} \Delta_{N}: a=\sum_{l=1}^{d} n_{l} N e_{l}, n_{l} \in \mathbb{Z} \text { such that } T_{a} \Delta_{N} \cap \Lambda \neq \emptyset\right\}
$$

and

$$
\Lambda_{N}^{-} \equiv\left\{\bigcup T_{a} \Delta_{N}: a=\sum_{l=1}^{d} n_{l} N e_{l}, n_{l} \in \mathbb{Z} \text { such that } T_{a} \Delta_{N} \subset \Lambda\right\} .
$$

The number of different cubes in the sum (1.9a) respectively (1.9b) is denoted by $n\left(\Lambda_{N}^{+}\right)$respectively $n\left(\Lambda_{N}^{-}\right)$.

A countable base $\mathscr{F}_{0}=\left\{\Lambda_{n} \in \mathscr{F}\right\}_{n \in \mathbb{N}}$ is called a van Hove sequence iff for any $N \in \mathbb{N}$

$$
\lim _{\mathscr{F}_{0}} \frac{\left|\Lambda_{N}^{-}\right|}{\left|\Lambda_{N}^{+}\right|}=1
$$

Let $(\Omega, \Sigma) \equiv(S, \mathscr{S})^{\mathbf{\Gamma}}$, with $(S, \mathscr{S})^{\mathbf{\Gamma}}$ being a standard Borel space, be the space of spin configurations $\sigma$. Typical examples of $(S, \mathscr{S})$ used in statistical mechanics are provided by: a) $S$ a finite set, b) $S \equiv \mathscr{S}^{N-1}$ a sphere in $\mathbb{R}^{N}$, with natural topologies respectively. A spin $\sigma_{i}$ at a site $i \in \Gamma$ is by definition the $i^{\text {th }}$ coordinate function on $(\Omega, \Sigma)$. For $\Lambda \subset \Gamma$ let $\Sigma_{\Lambda}$ be the smallest $\sigma$-algebra contained in $\Sigma$ such that all the 
functions $\left\{\sigma_{i}: i \in \Lambda\right\}$ are $\Sigma_{\Lambda}$-measurable. If a function $F$ is $\Sigma_{\Lambda}$-measurable we write $F \in \Sigma_{\Lambda}$.

By $\mu$ with various subscripts, we will denote probability measures on $(\Omega, \Sigma)$. A probability measure $\mu_{0}$, defined as a product of probability measures on $(S, \mathscr{P})$, is called a free measure. For $F \in \Sigma$, by $\mu(F)$ we denote the expectation value of $F$ computed with a measure $\mu$. For $F_{1}, F_{2} \in \Sigma$ a truncated correlation function is defined by

$$
\mu\left(F_{1}, F_{2}\right) \equiv \mu F_{1} F_{2}-\mu F_{1} \mu F_{2} .
$$

We introduce also another standard Borel space $(\mathbf{J}, \mathscr{J})$ to describe an additional randomness in the lattice system. Let $E$ be a probability measure on $(\mathbf{J}, \mathscr{J})$. We will use a similar convention as described above to denote the corresponding expectations. It will be assumed that one can distinguish a family $\left\{\mathscr{J}_{A}\right\}_{\Lambda \in \mathscr{F}}$ of sub- $\sigma$-algebras in $\mathscr{J}$, so that any functions $F_{1} \in \mathscr{J}_{\Lambda}, F_{2} \in \mathscr{J}_{\Lambda^{\prime}}$ are independent random variables on $(\mathbf{J}, \mathscr{J}, E)$ if $\Lambda \cap \Lambda^{\prime}=\emptyset$. Let

$$
\mathbf{T} \ni a \rightarrow T_{a}
$$

be a representation of the translation group by measurable bijections on $(\Omega, \Sigma)$ $\times(\mathbf{J}, \mathscr{J})$. In particular we define the representation of translations on $(\Omega, \Sigma)$ (implicitly) by requiring

$$
T_{a} \sigma_{i}=\sigma_{i-a} \text { for all } i \in \Gamma .
$$

We say that a probability measure $\mu$ on $(\Omega, \Sigma)$, respectively $E$ on $(\mathbf{J}, \mathscr{J})$, is translation invariant iff for any $F \in \Sigma$, we have

$$
T_{a}^{*} \mu(F) \equiv \mu\left(F\left(T_{-a} \sigma\right)\right)=\mu(F(\sigma))
$$

for all $a \in \mathbf{T}$, and similarly for $E$. Afterwards we will assume that the free measure $\mu_{0}$ and the measure $E$ are translationally invariant. For a sequence of functions $\left\{F_{A}\right\}_{\Lambda \in \mathscr{F}_{0}}$ we write $\lim _{\mathscr{F}_{0}} F_{A}$ to denote its $L_{1}(E)$ limit (if exists). Let $\mathbb{M}(\Omega \times \mathbf{J}, \mathbb{R})$ denote the space of jointly measurable functions on $(\Omega, \Sigma) \times(\mathbf{J}, \mathscr{J})$.

Definition 1.1. An interaction $\Phi$ is a function

$$
\Phi: \mathscr{F} \rightarrow \mathbb{M}(\Omega \times \mathbf{J}, \mathbb{R})
$$

satisfying for any $X \in \mathscr{F}$

$$
\Phi_{X} \in \Sigma_{X} \times \mathscr{J}_{X} .
$$

(Frequently it is useful to consider also complex valued interactions.) An interaction $\Phi$ satisfying for any $a \in \mathbf{T}$ the condition

$$
\Phi_{T_{a} X}(J, \sigma)=\Phi_{X}\left(T_{-a} J, T_{-a} \sigma\right)
$$

for all $X \in \mathscr{F}$ and any $J \in \mathbf{J}, \sigma \in \Omega$, is called translation invariant. An interaction $\Phi$ is of finite range $0 \leqq r(\Phi)<\infty$ iff for any $X \in \mathscr{F}$ with diam $X>r(\Phi)$ we have $\Phi_{X} \equiv 0$, and is of finite body type $0 \leqq \tau(\Phi)<\infty$ iff $\Phi_{X} \equiv 0$ for all $X \in \mathscr{F},|X|>\tau(\Phi)$.

As follows from Definition 1.1, the interactions form a linear space. For further purposes we would like to distinguish the following Banach spaces of interactions: The first one, denoted by $\mathbb{B}^{c l}(E)$, is a simple generalization of the classical space considered in $[54,38]$ and is defined by

$$
\mathbb{B}^{\mathrm{cl}}(E) \equiv\left\{\Phi:\|\Phi\|_{\mathrm{cl}}<\infty\right\},
$$


where we use the norm

$$
\|\Phi\|_{\mathrm{cl}}=\sup _{i \in \Gamma} \sum_{\substack{\boldsymbol{X} \in \mathscr{F} \\ i \in X}} \frac{1}{|X|} \sup _{\substack{\sigma \in \Omega \\ J \in \mathbf{J}}}\left|\Phi_{X}\right| .
$$

To include unbounded interactions we define a space

$$
\mathbb{B}^{u}(E) \equiv\left\{\Phi:\|\Phi\|_{u}<\infty\right\},
$$

where

$$
\|\Phi\|_{u}:=\sup _{i \in \Gamma} \sum_{\substack{X \in \mathscr{F} \\ i \in X}} \frac{1}{|X|} E\left(\sup _{\sigma \in \Lambda}\left|\Phi_{X}\right|\right) .
$$

From this definition we see that $\mathbb{B}^{\text {cl }}(E) \subset \mathbb{B}^{u}(E)$ and in general $\mathbb{B}^{u}(E) \backslash \mathbb{B}^{\text {cl }}(E)$ is nonempty. The above Banach spaces still do not exhaust all "good" interactions. To consider long range interactions, as those in (0.1)-(0.3), let us distinguish a class of interactions $\Phi$ satisfying the following conditions $(\mathrm{C})$ :

(Ci): For any $X, X^{\prime} \in \mathscr{F}, X \neq X^{\prime}$ and any $\sigma, \sigma^{\prime} \in \Omega$, the functions $\Phi_{X}(\cdot, \sigma)$ and $\Phi_{X^{\prime}}\left(\cdot, \sigma^{\prime}\right)$ are independent random variables on $(\mathbf{J}, \mathscr{J}, E)$.

(Cii): $\forall X \in \mathscr{F}, \sigma \in \Omega$,

$$
E \Phi_{X}(\cdot, \sigma)=0
$$

Then we define a Banach space

$$
\mathbb{B}^{w}(E) \equiv\left\{\Phi: \Phi \in(C) \text { and }\|\Phi\|_{w}<\infty\right\}
$$

where the norm is given by

$$
\|\Phi\|_{w}:=\sup _{i \in \Gamma}\left(\sum_{\substack{X \in \mathscr{F} \\ i \in X}} \frac{1}{|X|} E\left(\sup _{\sigma \in A}\left|\Phi_{X}\right|\right)^{2}\right)^{1 / 2} .
$$

As one can easily see we have in general

$$
\mathbb{B}^{u}(E) \cap \mathbb{B}^{w}(E) \neq \emptyset \quad \text { and } \quad \mathbb{B}^{u}(E) \div \mathbb{B}^{w}(E) \neq \emptyset,
$$

with $\div$ denoting the symmetric difference. (One may wish to introduce the corresponding Banach spaces $\mathbb{B}(E)$, suitable for discussion of Gibbs measures, by omitting the factors $\frac{1}{|X|}$ in the corresponding norms.) Note that the random-site long-range interactions $(0.4)$ and $(0.6)$ do not belong to $\mathbb{B}^{u}(E) \cup \mathbb{B}^{w}(E)$. Therefore we have to consider one more space of interactions. We would like to define it here as follows:

$$
\overline{\mathbb{B}(E)} \equiv\left\{\Phi: \sup _{\Lambda \in \mathscr{F}} \sup _{\sigma \in \Omega} \frac{1}{|\Lambda|}\left|\sum_{X \subset A} \Phi_{X}\right|<\infty, E-\text { a.e. }\right\} .
$$

It is clear that the systems with interactions from the class $\overline{\mathbb{B}(E)}$ behave thermodynamically well. However for some purposes this definition is too general. We will discuss it in Sect. 5. (For some short discussion concerning nonthermodynamically stable interactions, see [32].) If this will not cause confusion, we will write $\mathbb{B}(E)$ to denote the various space of interactions defined above or given as the sums of them. Then $\mathbb{B}_{I}(E)$ will mean the subspace of translation invariant interactions. 
For $\Lambda \in \mathscr{F}$ and $\Phi \in \mathbb{B}(E)$ we define a hamiltonian function by

$$
H_{\Lambda}(\Phi) \equiv \sum_{X \subseteq \Lambda} \Phi_{X}
$$

and a finite volume pressure

$$
p_{\Lambda}(\Phi) \equiv \frac{1}{|\Lambda|} \ln \mu_{0}\left(e^{-H_{\Lambda}(\Phi)}\right) .
$$

We wish to study the existence and properties of the infinite volume limit of the sequence of pressure functionals

$$
\mathbb{B}_{I}(E) \ni \Phi \mapsto p_{\Lambda}(\Phi), \quad \Lambda \in \mathscr{F}_{0} .
$$

\section{The Pressure Functional for Random Interactions I}

In this section we study a pressure functional defined on a Banach space

$$
\mathbb{B}_{I}(E)=\mathbb{B}_{I}^{u}(E)+\mathbb{B}_{I}^{w}(E) .
$$

The elements of this space are the pairs $\Phi \equiv(\phi, \psi)$, denoted also as $\Phi \equiv \phi+\psi$, with $\phi \in \mathbb{B}_{I}^{u}(E)$ and $\psi \in \mathbb{B}_{I}^{w}(E)$. It is assumed that the interactions $\phi \equiv\left\{\phi_{X}\right\}_{X \in \mathscr{F}}$ and $\psi$ $\equiv\left\{\psi_{X}\right\}_{X \in \mathscr{F}}$ are mutually independent in the sense that:

For any $X, X^{\prime} \in \mathscr{F}$ and any $\sigma, \sigma^{\prime} \in \Omega$ the random variables $\phi_{X}(\cdot, \sigma)$

and $\psi_{X^{\prime}}\left(\cdot, \sigma^{\prime}\right)$ defined on probability space $(\mathbf{J}, \mathscr{J}, E)$ are independent .

For $\Phi \in \mathbb{B}_{I}(E), \Phi=\phi+\psi$ we define a norm

$$
\|\Phi\|:=\|\phi\|_{u}+\|\psi\|_{w} .
$$

A hamiltonian function is given by

for any $\Lambda \in \mathscr{F}$.

$$
H_{\Lambda}(\Phi) \equiv \sum_{X \subset A} \Phi_{X} \equiv H_{\Lambda}(\phi)+H_{\Lambda}(\psi)
$$

Let $\mu_{0}$ be a translation invariant product probability measure on $(\Omega, \sigma)$. A finite volume pressure functional

$$
\mathbb{B}_{I}(E) \ni \Phi \mapsto p_{\Lambda}(\Phi), \quad \Lambda \in \mathscr{F}
$$

is defined by

$$
p_{\Lambda}(\Phi):=\frac{1}{|\Lambda|} \ln \mu_{0}\left(e^{-H_{\Lambda}(\Phi)}\right)
$$

The main result of this section is the following.

Theorem 2.1. Let $E$ be a translation invariant ergodic probability measure on $(\mathbf{J}, \mathscr{J})$. Then for any $\Phi \in \mathbb{B}_{I}(E)$ then infinite volume limit

$$
p(\Phi) \equiv \lim _{\mathscr{F}_{0}} p_{\Lambda}(\Phi)
$$

exists and is independent of $J \in \mathbf{J}, E$-a.e. and the van Hove sequence $\mathscr{F}_{0}$. Moreover the convex functional

$$
\mathbb{B}_{I}(E) \ni \Phi \rightarrow p(\Phi)
$$

satisfies the following continuity condition: 
For any $\Phi, \Phi^{\prime} \in \mathbb{B}(E), \Phi \equiv \phi+\psi$ and $\Phi^{\prime} \equiv \phi^{\prime}+\psi^{\prime}$

$$
\left|p(\Phi)-p\left(\Phi^{\prime}\right)\right| \leqq\left\|\phi-\phi^{\prime}\right\|_{u}+\max \left(\|\psi\|_{w},\left\|\psi^{\prime}\right\|_{w}\right)\left\|\psi-\psi^{\prime}\right\|_{w} .
$$

Remark. As follows from Definition (2.3) of the norm $\|\cdot\|$ in Banach space $\mathbb{B}_{I}(E)$, the right-hand side of (2.9) can be bounded by

$$
\text { r.h.s. }(2.9) \leqq\left[1+\max \left(\|\Phi\|,\left\|\Phi^{\prime}\right\|\right)\right]\left\|\Phi-\Phi^{\prime}\right\| \text {. }
$$

Proof. First we show that $\left\{p_{A}\right\}_{\Lambda \in \mathscr{F}_{0}}$ and $\left\{E p_{A}\right\}_{\Lambda \in \mathscr{F}_{0}}$ converge to the same limit and then we prove the continuity property (2.9) using the corresponding continuity of $E p_{A}, \Lambda \in \mathscr{F}_{0}$. Let $\Lambda \in \mathscr{F}_{0}$ be such that for some $N \in \mathbb{N}, \Lambda_{N}^{-}$defined in (1.9) is nonempty and let $n\left(\Lambda_{N}^{-}\right)$be the number of cubes $\left\{\Delta_{N}^{(l)} \equiv T_{a_{i}} \Delta_{N}: a_{\imath} \equiv \sum_{k=1}^{d} n_{k} N e_{k}\right\}$ satisfying

$$
\left.\begin{array}{rl}
\Delta_{N}^{(l)} \cap \Delta_{N}^{\left(i^{\prime}\right)} & =\emptyset \text { if } \quad l \neq l^{\prime} \\
\Lambda_{N}^{-} & =\bigcup_{l} \Delta_{N}^{(l)}
\end{array}\right\} .
$$

By the fundamental theorem of calculus we have

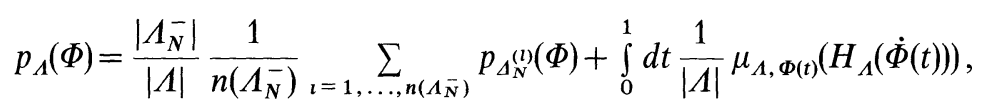

where for $t \in[0,1]$ we defined $\Phi(t)$ by

$$
\Phi_{X}(t):=\left\{\begin{array}{l}
\Phi_{X} \text { if } X \cong \Delta_{N}^{(l)} \text { for some } i=1, \ldots, n\left(\Lambda_{N}^{-}\right) \\
t \Phi_{X} \text { otherwise }
\end{array}\right.
$$

we set $\dot{\Phi}(t) \equiv \frac{d}{d t} \Phi(t)$, and

$$
\mu_{\Lambda, \Phi(t)}(\cdot) \equiv \frac{\mu_{0}\left(e^{-H_{\Lambda}(\Phi(t))} \cdot\right)}{\mu_{0}\left(e^{-H_{\Lambda}(\Phi(t))}\right)} .
$$

We will need to estimate the second term on the right-hand side of (2.12). Using the representation

$$
\Phi(t)=\phi(t)+\psi(t)
$$

we write

$$
\frac{1}{|\Lambda|} \mu_{\Lambda, \Phi(t)}\left(H_{\Lambda}(\dot{\Phi}(t))\right)=\frac{1}{|\Lambda|} \mu_{\Lambda, \Phi(t)} H_{\Lambda}(\dot{\phi}(t))+\frac{1}{|\Lambda|} \mu_{\Lambda, \Phi(t)} H_{\Lambda}(\dot{\psi}(t))
$$

and bound the $L_{1}(E)$ norms of the terms from the right-hand side of (2.16) separately. The result is given in the following lemma in which we use the notation introduced above.

Lemma 2.2. Let $L(N) \in \mathbb{N}$ be a function of $N \in \mathbb{N}$ such that $L(N) / N \rightarrow 0$ and $L(N)$ $\rightarrow \infty$ as $N \rightarrow \infty$. There is a constant $0<C<\infty$ (independent of $\Lambda \in \mathscr{F}_{0}$ and $\Phi \in \mathbb{B}(E)$ ) and a positive function $\varepsilon_{\phi}(L(N))$ converging to zero as $L(N) \rightarrow \infty$, such that for any $\Lambda \in \mathscr{F}_{0}$,

$$
E\left|\frac{1}{|\Lambda|} \mu_{\Lambda, \Phi(t)} H_{\Lambda}(\dot{\phi}(t))\right| \leqq\left(\frac{\left|\Lambda \backslash \Lambda_{N}^{-}\right|}{|\Lambda|}+C \frac{L(N)}{N}\right)\|\phi\|_{u}+\varepsilon_{\phi}(L(N)) .
$$


Moreover there is a positive function $\tilde{\varepsilon}_{\psi}(L(N))$, converging to zero as $L(N) \rightarrow \infty$, such that for any $\Lambda \in \mathscr{F}_{0}$,

$$
E\left|\frac{1}{|\Lambda|} \mu_{\Lambda, \Phi(t)} H_{\Lambda}(\dot{\psi}(t))\right| \leqq 2\left(\frac{\left|\Lambda \backslash \Lambda_{N}^{-}\right|}{|\Lambda|}+C \frac{L(N)}{N}\right)\|\psi\|_{w}^{2}+2 \tilde{\varepsilon}_{\psi}(L(N))+\frac{1}{|\Lambda|^{1 / 2}}\|\psi\|_{w} .
$$

Remark. The functions $\varepsilon_{\phi}(L(N))$ and $\tilde{\varepsilon}_{\psi}(L(N))$ are explicitly given below by $(2.42)$ and respectively (2.52).

Now using Lemma 2.2 and formula (2.12) we prove the convergence of the sequence $\left\{E p_{\Lambda}(\Phi)\right\}_{\Lambda \in \mathscr{F}_{0}}$. To do this, it is enough to show that the considered sequence has the Cauchy property. Let us take a number $0<\varepsilon$. Then using (2.12), translation invariance of the interaction $\Phi$ and of the measure $E$ we get, for any $\Lambda_{k}$, $\Lambda_{m} \in \mathscr{F}_{0}$ :

$$
\begin{aligned}
\left|E p_{\Lambda_{k}}(\Phi)-E p_{\Lambda_{m}}(\Phi)\right| \leqq & \left|\frac{\left|\left(\Lambda_{k}\right)_{N}^{-}\right|}{\left|\Lambda_{k}\right|}-\frac{\left|\left(\Lambda_{m}\right)_{N}^{-}\right|}{\left|\Lambda_{m}\right|}\right|\left|E p_{\Delta_{N}}(\Phi)\right| \\
& +\int_{0}^{1} d t E\left|\frac{1}{\left|\Lambda_{k}\right|} \mu_{\Lambda_{k}, \Phi(t)}\left(H_{\Lambda_{k}}(\dot{\Phi}(t))\right)\right| \\
& +\int_{0}^{1} d t E\left|\frac{1}{\left|\Lambda_{m}\right|} \mu_{\Lambda_{m}, \Phi(t)}\left(H_{\Lambda_{m}}(\dot{\Phi}(t))\right)\right|
\end{aligned}
$$

Since the sequence $\mathscr{F}_{0} \equiv\left\{\Lambda_{n} \in \mathscr{F}\right\}_{n \in \mathbb{N}}$ is van Hove and, as follows from Lemma 2.3 given below, we have for any $N \in \mathbb{N}$,

$$
\left|E p_{\Delta_{N}}(\Phi)\right| \leqq\|\phi\|_{u}+\|\psi\|_{w}^{2},
$$

so there is $n_{0} \in \mathbb{N}$ such that for any $k, m \geqq n_{0}$,

$$
\left|\frac{\left|\left(\Lambda_{k}^{-}\right)_{N}\right|}{\left|\Lambda_{k}\right|}-\frac{\left|\left(\Lambda_{m}^{-}\right)_{N}\right|}{\left|\Lambda_{m}\right|}\right|\left|E p_{\Delta_{N}}(\Phi)\right|<\frac{\varepsilon}{2} .
$$

From Lemma 2.2 the second and third term on the right-hand side of (2.19) are bounded by

$$
\begin{aligned}
\mathcal{O}\left(n_{0}, N\right) \equiv & \max _{\Lambda \in \mathscr{F}, \Lambda_{n_{0}} \subset \Lambda}\left\{\frac{\left|\Lambda \backslash \Lambda_{N}^{-}\right|}{|\Lambda|}\left(\|\phi\|_{u}+2\|\psi\|_{w}^{2}\right)+\frac{1}{|\Lambda|^{1 / 2}}\|\psi\|_{w}\right\} \\
& +\frac{L(N)}{N} C\left(\|\phi\|_{u}+2\|\psi\|_{w}^{2}\right)+\varepsilon_{\phi}(L(N))+2 \tilde{\varepsilon}_{\psi}(L(N)) .
\end{aligned}
$$

Since $\mathscr{F}_{0}$ is a van Hove sequence, we can find some $N_{0} \in \mathbb{N}$ and $m_{0} \in \mathbb{N}$ such that

$$
2 \mathcal{O}\left(n_{0}, N\right)<\frac{\varepsilon}{2}
$$

for all $N \geqq N_{0}$ and $n_{0} \geqq m_{0}$. This together with (2.21) shows that

$$
\left|E p_{\Lambda_{k}}(\Phi)-E p_{\Lambda_{m}}(\Phi)\right|<\varepsilon
$$

for all $\Lambda_{k}, \Lambda_{m} \in \mathscr{F}_{0}, k, m \geqq m_{0}$ with some sufficiently big $m_{0} \in \mathbb{N}$, i.e. we have the desired Cauchy property. Therefore the limit of the sequence $\left\{E p_{\Lambda}(\Phi)\right\}_{\Lambda \in \mathscr{F}_{0}}$ exists and, as follows easily from the above considerations, is independent of the van Hove sequence $\mathscr{F}_{0}$. (In particular one does not need to assume that $\mathscr{F}_{0}$ is strictly 
increasing.) To show that $\left\{p_{\Lambda}(\Phi)\right\}_{\Lambda \in \mathscr{F}}$ converges to the same limit, let us note that using the triangle inequality, we get

$$
\begin{aligned}
\left|p_{\Lambda}-E p_{\Lambda}\right| \leqq & \left|p_{\Lambda}-\frac{\left|\Lambda_{N}^{-}\right|}{|\Lambda|} \frac{1}{n\left(\Lambda_{N}^{-}\right)} \sum_{\imath=1, \ldots, n\left(\Lambda_{\bar{N}}\right)} p_{\Delta_{N}^{(l)}}\right| \\
& +\left|\frac{\left|\Lambda_{N}^{-}\right|}{|\Lambda|}-1\right| \cdot\left|E p_{\Delta_{N}}\right| \\
& +\mid \frac{1}{n\left(\Lambda_{N}^{-}\right)} \sum_{\imath=1, \ldots, n\left(\Lambda_{\bar{N}}\right)} p_{\Delta_{N}^{(l)}-E p_{\Delta_{N}} \mid} \\
& +\left|E p_{\Lambda}-E p_{\Delta_{N}}\right| .
\end{aligned}
$$

Using Lemma 2.2 we see that

$$
\lim _{N \rightarrow \infty} \lim _{\mathscr{F}_{0}} E\left|p_{\Lambda}-\frac{\left|\Lambda_{N}^{-}\right|}{|\Lambda|} \frac{1}{n\left(\Lambda_{N}^{-}\right)} \sum_{\imath=1, \ldots, n\left(\Lambda_{\bar{N}}\right)} p_{\Delta_{N}^{(l)}}\right|=0 .
$$

Since $\mathscr{F}_{0}$ is van Hove, so using (2.20) (Lemma 2.3 below) we get

$$
\lim _{\mathscr{F}_{0}}\left|\frac{\left|\Lambda_{N}^{-}\right|}{|\Lambda|}-1\right|\left|E p_{\Delta_{N}}\right|=0 \text {. }
$$

From the fact that $\lim _{\mathscr{F}_{0}} E p_{\Lambda}(\Phi)$ exists and is independent of the van Hove sequence chosen, we get

$$
\lim _{N \rightarrow \infty} \lim _{\mathscr{F}_{0}}\left|E p_{\Lambda}-E p_{\Delta_{N}}\right|=0 .
$$

Finally, using the ergodicity of the translation invariant measure $E$, Lemma 2.3 and the ergodic theorem for the commutative group of translations (see e.g. [42]), we get

$$
\lim _{\mathscr{F}_{0}}\left(\frac{1}{n\left(\Lambda_{N}^{-}\right)} \sum_{\imath=1, \ldots, n\left(\Lambda_{\bar{N}}\right)} p_{\Delta_{N}}-E p_{\Delta_{N}}\right)=0,
$$

$E$-a.e. and so, using Lemma 2.3, also in $L_{1}(E)$. Combining (2.25) and (2.26)-(2.29) we conclude that

$$
\lim _{\mathscr{F}_{0}} E\left|p_{\Lambda}(\Phi)-E p_{\Lambda}(\Phi)\right|=0
$$

i.e.

$$
p(\Phi) \equiv \lim _{\mathscr{F}_{0}} p_{\Lambda}(\Phi)=\lim _{\mathscr{F}_{0}} E p_{\Lambda}(\Phi)
$$

exist and is independent of the van Hove sequence $\mathscr{F}_{0}$. The formula (2.31) defines a (nonrandom) functional,

$$
\mathbb{B}_{I}(E) \ni \Phi \mapsto p(\Phi) \in \mathbb{R}
$$

which (as follows easily by Hölder's inequality) is convex, i.e. for any $s \in[0,1]$ and any $\Phi, \Phi^{\prime} \in \mathbb{B}_{I}(E)$

$$
p\left(s \Phi+(1-s) \Phi^{\prime}\right) \leqq s p(\Phi)+(1-s) p\left(\Phi^{\prime}\right) .
$$

The continuity property (2.9) of the functional $p(\Phi)$ follows from the following lemma:

Lemma 2.3. Let $\Phi, \Phi^{\prime} \in \mathbb{B}_{I}(E), \Phi \equiv \phi+\psi, \Phi^{\prime} \equiv \phi^{\prime}+\psi^{\prime}$. Then for any $\Lambda \in \mathscr{F}$,

$$
\left|E p_{\Lambda}(\Phi)-E p_{\Lambda}\left(\Phi^{\prime}\right)\right| \leqq\left\|\phi-\phi^{\prime}\right\|_{u}+\max \left(\|\psi\|_{w},\left\|\psi^{\prime}\right\|_{w}\right)\left\|\psi-\psi^{\prime}\right\|_{w} .
$$


Moreover

$$
E\left|p_{\Lambda}(\Phi)\right| \leqq\|\phi\|_{u}+2\|\psi\|_{w}+o\left(|\Lambda|^{-1 / 2}\right) .
$$

Proof of Lemma 2.2. We begin with the proof of (2.17). Using the definition of $\phi(t)$ given by (2.13) and (2.15), we have

$$
E\left|\frac{1}{|\Lambda|} \mu_{\Lambda, \Phi(t)} H_{\Lambda}(\dot{\phi}(t))\right| \leqq \frac{1}{|\Lambda|} \sum_{\substack{X \subset \Delta_{N}^{(2)}, \underline{X}=1, \ldots, i \in X, n(\Lambda \bar{N})}} E \sup _{\sigma \in \Omega}\left|\phi_{X}\right| .
$$

The right-hand side of (2.35) can be written in the form

$$
\begin{aligned}
\text { r.h.s. (2.35) } & =\frac{1}{|\Lambda|} \sum_{X \subset \Lambda \backslash \Lambda \bar{N}} E \sup _{\sigma \in \Omega}\left|\phi_{X}\right|+\frac{1}{|\Lambda|} \sum_{i=1}^{n(\Lambda \bar{N})} \sum_{i \in \Delta_{N}^{(l)}} \sum_{\substack{x \subseteq \Lambda, i \in X \\
\bar{X} \not \Delta \Delta_{N}^{(l)}}} \frac{1}{|X|} E \sup _{\sigma \in \Omega}\left|\phi_{X}\right| \\
& \equiv A(2.36)+B(2.36) .
\end{aligned}
$$

The first term from the right-hand side of (2.36) satisfies the estimate

$$
A(2.36) \leqq \frac{\left|\Lambda \backslash \Lambda_{N}^{-}\right|}{|\Lambda|}\|\phi\|_{u} .
$$

Since by our assumptions the interaction $\phi$ and the measure are translation invariant, the term $B(2.36)$ is bounded by

$$
\begin{aligned}
B(2.36) & \leqq \frac{1}{\left|\Delta_{N}\right|} \sum_{i \in \Delta_{N}} \sum_{\substack { X \in \mathscr{F} \\
\begin{subarray}{c}{X \in \Delta_{N} \\
i \in X{ X \in \mathscr { F } \\
\begin{subarray} { c } { X \in \Delta _ { N } \\
i \in X } }\end{subarray}} \frac{1}{|X|} E \sup _{\sigma \in \Omega}\left|\phi_{X}\right| \\
& \equiv A(2.38)+B(2.38),
\end{aligned}
$$

where for some $L \equiv L(N) \in \mathbb{N}$ we introduce the following partition of the right-hand side of (2.38):

$$
A(2.38) \equiv \frac{1}{\left|\Delta_{N}\right|} \sum_{\substack{i \in \Delta_{N} \\
d\left(i, \partial \Delta_{N}\right) \leqq L(N)}} \sum_{\substack { X \in \mathscr{F} \\
\begin{subarray}{c}{\mathcal{F}_{N} \\
i \in X{ X \in \mathscr { F } \\
\begin{subarray} { c } { \mathcal { F } _ { N } \\
i \in X } }\end{subarray}} \frac{1}{|X|} E \sup _{\sigma \in \Omega}\left|\phi_{X}\right|
$$

and

$$
B(2.38) \equiv \frac{1}{\left|\Delta_{N}\right|} \sum_{\substack{i \in \Delta_{N} \\ d\left(i, \partial \Delta_{N}\right)>L(N)}} \sum_{\substack{X \in \mathscr{F} \\ X \in \Delta_{N} \\ i \in X}} \frac{1}{|X|} E \sup _{\sigma \in \Omega}\left|\phi_{X}\right| .
$$

The expression $A(2.38)$ satisfies the bound

$$
\begin{aligned}
A(2.38) & \leqq \frac{1}{\left|\Delta_{N}\right|}\left|\left\{i \in \Delta_{N}: d\left(i, \partial \Delta_{N}\right) \leqq L(N)\right\}\right| \cdot\|\phi\|_{u} \\
& \leqq C\|\phi\|_{u} \frac{L(N)}{N}
\end{aligned}
$$

with some constant $0<C<\infty$ independent of $\phi \in \mathbb{B}^{u}(E)$ and $L(N), N \in \mathbb{N}$. Using the translation invariance of $\phi$ and $E$ we bound $B(2.38)$ by

$$
B(2.38) \leqq \sum_{\substack{X \in \mathscr{F} \\ 0 \in X, \operatorname{diam} X>L(N)}} \frac{1}{|X|} E \sup _{\sigma \in \Omega}\left|\phi_{X}\right| \equiv \varepsilon_{\phi}(L(N)) .
$$


Note that from the assumption that $\|\phi\|_{u}<\infty$ it follows that the function $\varepsilon_{\phi}(L(N))$ goes to zero as $L(N) \rightarrow \infty$. Combining $(2.39)-(2.42)$ we get

$$
B(2.36) \leqq C\|\phi\|_{u} \frac{L(N)}{N}+\varepsilon_{\phi}(L(N)) .
$$

From this, (2.37) and (2.35)-(2.36) we get

$$
E\left|\frac{1}{|\Lambda|} \mu_{\Lambda, \Phi(t)} H_{\Lambda}(\dot{\phi}(t))\right| \leqq\left(\frac{\left|\Lambda \backslash \Lambda_{N}^{-}\right|}{|\Lambda|}+C \frac{L(N)}{N}\right)\|\phi\|_{u}+\varepsilon_{\phi}(L(N)) .
$$

This ends the proof of (2.17).

We shall now prove (2.18). By definition of $\psi(t)$ in (2.13) and (2.15) we have

$$
\frac{1}{|\Lambda|} \mu_{\Lambda, \Phi(t)} H_{\Lambda}(\dot{\psi}(t))=\frac{1}{|\Lambda|} \sum_{\substack{X \subseteq \Lambda, i \in X \\ X \notin \Delta_{N}, l=1, \ldots, n\left(\Lambda_{\bar{N}}\right)}} \mu_{\Lambda, \Phi(t)}\left(\psi_{X}\right) .
$$

Each term on right-hand side (2.45) we write, using a Taylor expansion, in the form

$$
\mu_{\Lambda, \Phi(t)}\left(\psi_{X}\right)=\mu_{\Lambda, \Phi\left(t, s_{X}=0\right)}\left(\psi_{X}\right)-t \int_{0}^{1} d s_{X} \mu_{\Lambda, \Phi\left(t, s_{X}\right)}\left(\psi_{X}, \psi_{X}\right),
$$

where $\Phi\left(t, s_{X}\right), s_{X} \in[0,1]$ is defined by

$$
\Phi\left(t, s_{X}\right)_{Y}:=\left\{\begin{array}{lll}
\Phi(t)_{Y} & \text { for } & Y \neq X \\
\phi_{X}(t)+s_{X} \psi_{X}(t) & \text { for } & Y=X
\end{array} .\right.
$$

Inserting (2.46) into (2.45), we get the following estimate for the $L_{1}(E)$ norm of (2.45):

$$
E\left|\frac{1}{|\Lambda|} \mu_{\Lambda, \Phi(t)} H_{\Lambda}(\dot{\psi}(t))\right| \leqq A(2.48)+B(2.48)
$$

with

$$
A(2.48) \equiv E\left|\frac{1}{|\Lambda|} \sum_{X \cong \Lambda}^{\prime} \mu_{\Lambda, \Phi\left(t, s_{X}=0\right)}\left(\psi_{X}\right)\right|
$$

and

$$
B(2.48) \equiv E\left|\frac{1}{|\Lambda|} \sum_{X \subseteq \Lambda}^{\prime} \int_{0}^{1} d s_{X} \mu_{\Lambda, \Phi\left(t, s_{X}\right)}\left(\psi_{X}, \psi_{X}\right)\right|,
$$

where $\sum_{X \subseteq A}^{\prime}$ means restricted summation over $X \subseteq \Lambda$ as on the right-hand side of (2.45). The term $B(2.48)$ is easy to estimate. We have

$$
B(2.48) \leqq \frac{1}{|\Lambda|} \sum_{X \subseteq \Lambda}^{\prime} E\left(\sup _{\sigma \in \Omega}\left|\psi_{X}\right|\right)^{2} .
$$

Since the right hand side of (2.51) is of the same structure as the right-hand side of (2.35), we can use the same arguments to get a similar bound as in (2.17), with $\|\psi\|_{w}^{2}$ instead of $\|\phi\|_{u}$ and the corresponding function

$$
\tilde{\varepsilon}_{\psi}(L(N)) \equiv \sum_{\substack{X \in \mathscr{F} \\ 0 \in X, \operatorname{diam} X>L(N)}} \frac{1}{|X|} E\left(\sup _{\sigma \in \Omega}\left|\psi_{X}\right|\right)^{2}
$$

instead of $\varepsilon_{\phi}(L(N))$. 
To estimate $A(2.48)$ we use Hölder's inequality and find a bound on the $L_{2}(E)$ norm of the interesting us integrant. First we write

$$
\begin{aligned}
& \left|\frac{1}{|\Lambda|} \sum_{Y \subseteq \Lambda}^{\prime} \mu_{\Lambda, \Phi\left(t, s_{X}=0\right)}\left(\psi_{X}\right)\right|^{2} \\
& \quad=\frac{1}{|\Lambda|^{2}} \sum_{X \subseteq \Lambda}^{\prime} \sum_{Y \subseteq \Lambda}^{\prime} \mu_{\Lambda, \Phi\left(t, s_{X}=0\right)}\left(\psi_{X}\right) \mu_{\Lambda, \Phi\left(t, s_{Y}=0\right)}\left(\psi_{Y}\right) \\
& \quad \equiv A(2.53)+B(2.53)
\end{aligned}
$$

with

$$
A(2.53) \equiv \frac{1}{|\Lambda|^{2}} \sum_{X \subseteq \Lambda}^{\prime}\left(\mu_{\Lambda, \Phi\left(t, s_{X}=0\right)} \psi_{X}\right)^{2}
$$

and

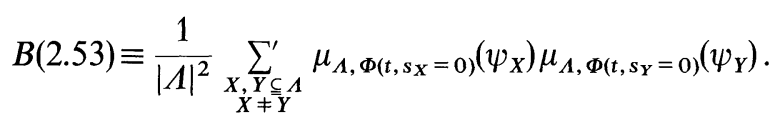

For the $E$ integral of (2.54) we have the bound

$$
E A(2.53) \leqq \frac{1}{|\Lambda|^{2}} \sum_{X \subseteq A}^{\prime} E\left(\sup _{\sigma \in \Omega}\left|\psi_{X}\right|\right)^{2} \leqq \frac{1}{|\Lambda|}\|\psi\|_{w}^{2} .
$$

To estimate the $E$ integral of (2.55) first we expand each term as follows

$$
\begin{aligned}
& \mu_{\Lambda, \Phi\left(t, s_{X}=0\right)}\left(\psi_{X}\right) \mu_{\Lambda, \Phi\left(t, s_{Y}=0\right)}\left(\psi_{Y}\right) \\
& =\left(\mu_{\Lambda \Phi\left(t, s_{X}=0, s_{Y}=0\right)}\left(\psi_{X}\right)-t \int_{0}^{1} d s_{Y} \mu_{\Lambda \Phi\left(t, s_{X}=0, s_{Y}\right)}\left(\psi_{X}, \psi_{Y}\right)\right) \\
& \quad \times\left(\mu_{\Lambda \Phi\left(t, s_{X}=0, s_{Y}=0\right)}\left(\psi_{Y}\right)-t \int_{0}^{1} d s_{X} \mu_{\Lambda \Phi\left(t, s_{Y}=0, s_{X}\right)}\left(\psi_{Y}, \psi_{X}\right)\right),
\end{aligned}
$$

where for $X \neq Y$ we defined

$$
\Phi\left(t, s_{X}, s_{Y}\right)_{Z} \equiv\left\{\begin{array}{ccc}
\Phi(t)_{Z} & \text { for } & Z \neq X, Y \\
\phi_{X}(t)+s_{X} \psi_{X}(t) & \text { for } & Z=X \\
\phi_{Y}(t)+s_{Y} \psi_{Y}(t) & \text { for } & Z=Y
\end{array} .\right.
$$

Now using the independence of the $\left\{\psi_{X}\right\}_{X \in \mathscr{F}}$ variables and the condition $E \psi_{X}=0$, $X \in \mathscr{F}$ from the definition of the space $\mathbb{B}^{w}(E)$ (and independence of $\phi$ 's and $\psi$ 's) we see that for $X \neq Y$,

$$
\begin{aligned}
& E \mu_{\Lambda, \Phi\left(t, s_{X}=0\right)}\left(\psi_{X}\right) \mu_{\Lambda, \Phi\left(t, s_{Y}=0\right)}\left(\psi_{Y}\right) \\
& \quad=t^{2} \int_{0}^{1} d s_{X}^{\prime} \int_{0}^{1} d s_{Y}^{\prime} E\left(\mu_{\Lambda, \Phi\left(t, s_{X}=0, s_{Y}^{\prime}\right)}\left(\psi_{X}, \psi_{Y}\right) \mu_{\Lambda, \Phi\left(t, s_{X}^{\prime}, s_{Y}=0\right)}\left(\psi_{Y}, \psi_{X}\right)\right) .
\end{aligned}
$$

The absolute value of (2.59) satisfies the bound

$$
|(2.59)| \leqq E\left(\sup _{\sigma \in \Omega}\left|\psi_{X}\right|\right)^{2} \cdot E\left(\sup _{\sigma \in \Omega}\left|\psi_{Y}\right|\right)^{2},
$$

where we used the independence of the $\psi_{X}$ and $\psi_{Y}$ variables for $X \neq Y$.

Using (2.57)-(2.59) we get

$$
|E B(2.53)| \leqq\left[\frac{1}{|\Lambda|} \sum_{X \subseteq \Lambda}^{\prime} E\left(\sup _{\sigma \in \Omega}\left|\psi_{X}\right|\right)^{2}\right]^{2}
$$


This together with (2.56) and (2.53), by use of Hölder's inequality, gives us the bound

$$
\begin{aligned}
A(2.48) & \leqq(E A(2.53)+E B(2.53))^{1 / 2} \\
& \leqq \frac{1}{|\Lambda|^{1 / 2}}\|\psi\|_{w}+\frac{1}{|\Lambda|} \sum_{X \leqq A}^{\prime} E\left(\sup _{\sigma \in \Omega}\left|\psi_{X}\right|\right)^{2} .
\end{aligned}
$$

The second term on the right-hand side of (2.62) is the same as the one used to estimate $B(2.48)$ in (2.51) and so satisfies the same bound as discussed before. Adding the bounds obtained for $B(2.48)$ and $A(2.48)$ we get (2.18). This ends the proof of Lemma 2.2.

Proof of Lemma 2.3. Let $\Phi, \Phi^{\prime} \in \mathbb{B}(E), \Phi \equiv \phi+\psi$ and $\Phi^{\prime} \equiv \phi^{\prime}+\psi^{\prime}$. For $t \in[0,1]$ we define

$$
\Phi(t) \equiv \phi(t)+\psi(t)
$$

with

$$
\phi(t) \equiv t \phi+(1-t) \phi^{\prime}, \quad \psi(t) \equiv t \psi+(1-t) \psi^{\prime} .
$$

For $\Lambda \in \mathscr{F}$, let us define

$$
\mu_{\Lambda, \Phi(t)}(\cdot):=\frac{\mu_{0}\left(e^{-H_{\Lambda}(\Phi(t))}\right)}{\mu_{0}\left(e^{-H_{\Lambda}(\Phi(t))}\right)} .
$$

Then we have

$$
p_{\Lambda}(\Phi)-p_{\Lambda}\left(\Phi^{\prime}\right)=-\int_{0}^{1} d t \frac{1}{|\Lambda|} \mu_{\Lambda, \Phi(t)}\left(H_{\Lambda}\left(\phi-\phi^{\prime}\right)\right)-\int_{0}^{1} d t \frac{1}{|\Lambda|} \mu_{\Lambda, \Phi(t)}\left(H_{\Lambda}\left(\psi-\psi^{\prime}\right)\right) .
$$

The absolute value of the expectation of the first term from the right-hand side of (2.66) satisfies the following estimate:

$$
E\left|\int_{0}^{1} d t \frac{1}{|\Lambda|} \mu_{\Lambda, \Phi(t)}\left(H_{\Lambda}\left(\phi-\phi^{\prime}\right)\right)\right| \leqq\left\|\phi-\phi^{\prime}\right\|_{u},
$$

where we used the definition (1.21) of the norm $\|\cdot\|_{u}$ together with some standard arguments.

To estimate the absolute value of expectation of the second term from the righthand side of (2.66) we use (Fubini's theorem and) the following equality based on a Taylor expansion

$$
\begin{aligned}
\frac{1}{|\Lambda|} \mu_{\Lambda, \Phi(t)} H_{\Lambda}\left(\psi-\psi^{\prime}\right)= & \frac{1}{|\Lambda|} \sum_{X \subseteq \Lambda} \mu_{\Lambda, \Phi(t)}\left(\psi_{X}-\psi_{X}^{\prime}\right) \\
= & \frac{1}{|\Lambda|} \sum_{X \subseteq \Lambda} \mu_{\Lambda, \Phi\left(t, s_{X}=0\right)}\left(\psi_{X}-\psi_{X}^{\prime}\right) \\
& -\frac{1}{|\Lambda|} \sum_{X \subseteq \Lambda} \int_{0}^{1} d s_{X} \mu_{\Lambda, \Phi\left(t, s_{X}\right)}\left(\psi_{X}-\psi_{X}^{\prime}, \psi_{X}(t)\right) \\
\equiv & A(2.68)+B(2.68),
\end{aligned}
$$

where for $s_{X} \in[0,1]$ we defined

$$
\Phi\left(t, s_{X}\right) \equiv\left\{\begin{array}{lll}
\Phi_{Y}(t) & \text { for } & Y \neq X \\
\phi_{X}(t)+s_{X} \psi(t) & \text { for } & Y=X
\end{array} .\right.
$$


Using the condition $(C)$ from the definition of $\psi \in \mathbb{B}^{w}(E)$ interactions and their independence of $\phi \in \mathbb{B}^{u}(E)$ we see that

$$
E(A(2.68))=0 \text {. }
$$

To estimate the absolute value of $B(2.68)$ we use the inequality

$$
\left|\mu_{\Lambda, \Phi(t)}\left(\psi_{X}-\psi_{X}^{\prime}, \psi_{X}(t)\right)\right| \leqq\left(\sup _{\sigma \in \Omega}\left|\psi_{X}-\psi_{X}^{\prime}\right|\right) \cdot\left(\sup _{\sigma \in \Omega}\left|\psi_{X}(t)\right|\right)
$$

(which follows from the Schwartz inequality by simple arguments). Using (2.71), translation invariance of the interaction and the measure $E$ together with Hölder's inequality we get

$$
\begin{aligned}
E|B(2.68)| & \leqq \frac{1}{|\Lambda|} \sum_{X \subseteq A} E\left(\sup _{\sigma \in \Omega}\left|\psi_{X}-\psi_{X}^{\prime}\right| \sup _{\sigma \in \Omega}\left|\psi_{X}(t)\right|\right) \\
& \leqq \sum_{\substack{X \in \mathscr{F} \\
0 \in X}} \frac{1}{|X|} E\left(\sup _{\sigma \in \Omega}\left|\psi_{X}-\psi_{X}^{\prime}\right| \sup _{\sigma \in \Omega}\left|\psi_{X}(t)\right|\right) \\
& \leqq\left\|\psi-\psi^{\prime}\right\|_{w} \cdot\|\psi(t)\|_{w} \\
& \leqq \max \left(\|\psi\|_{w},\left\|\psi^{\prime}\right\|_{w}\right)\left\|\psi-\psi^{\prime}\right\|_{w} .
\end{aligned}
$$

This together with (2.70) gives the estimate on (2.68), the expectation of the second sum from the right-hand side of (2.66). Combining this estimate with estimate (2.67) we get (2.34). This ends the proof of the continuity (2.34).

To show (2.34a), let us set $\Phi^{\prime} \equiv 0$ in (2.62)-(2.72). Using (2.66) together with (2.67) and (2.68)-(2.72) we see that to finish the proof we need only to estimate $E|A(2.68)|$. This is because the expectation of the absolute value of the second term on the right-hand side of (2.68) satisfies the following simple estimate:

$$
E|B(2.68)| \leqq\|\psi\|_{w}^{2} .
$$

Let us now note that we have

$$
\begin{aligned}
& E|A(2.68)|^{2}=\frac{1}{|\Lambda|^{2}} \sum_{X \cong \Lambda} E\left(\mu_{\Lambda, \Phi\left(t, s_{X}=0\right)} \psi_{X}\right)^{2} \\
& +\frac{1}{|\Lambda|^{2}} \sum_{\substack{X, Y \subseteq \Lambda \\
X \neq Y}} E \mu_{\Lambda, \Phi\left(t, s_{X}=0\right)} \psi_{X} \cdot \mu_{\Lambda, \Phi\left(t, s_{Y}=0\right)} \psi_{Y}=A(2.74)+B(2.74) .
\end{aligned}
$$

The first term from the right-hand side of (2.74) has the following simple estimate:

$$
E(A(2.74)) \leqq \frac{1}{|\Lambda|}\|\psi\|_{w}^{2}
$$

To estimate $B(2.74)$ we note that for $X, Y \subseteq \Lambda, X \neq Y$ we have

$$
\begin{aligned}
& \mu_{\Lambda, \Phi\left(t, s_{X}=0\right)} \psi_{X} \mu_{\Lambda, \Phi\left(t, s_{Y}=0\right)} \psi_{Y}=\left\{\mu_{\Lambda, \Phi\left(t, s_{X}, s_{Y}=0\right)} \psi_{X} \mu_{\Lambda, \Phi\left(t, s_{X}, s_{Y}=0\right)} \psi_{Y}\right. \\
& +\int_{0}^{1} d s_{Y}^{\prime} \mu_{\Lambda, \Phi\left(t, s_{X}=0, s_{Y}^{\prime}\right)}\left(\psi_{X}, \psi_{Y}\right) \mu_{\Lambda, \Phi\left(t, s_{X}, s_{Y}=0\right)} \psi_{Y} \\
& \left.+\mu_{\Lambda, \Lambda\left(t, s_{X}, s_{Y}=0\right)} \psi_{X} \int_{0}^{1} d s_{X}^{\prime} \mu_{\Lambda, \Phi\left(t, s_{Y}=0, s_{X}^{\prime}\right)}\left(\psi_{Y}, \psi_{X}\right)\right\} \\
& +\int_{0}^{1} d s_{Y}^{\prime} \mu_{\Lambda, \Phi\left(t, s_{X}=0, s_{Y}^{\prime}\right)}\left(\psi_{X}, \psi_{Y}\right) \int_{0}^{1} d s_{X}^{\prime} \mu_{\Lambda, \Phi\left(t, s_{Y}=0, s_{X}^{\prime}\right)}\left(\psi_{Y}, \psi_{X}\right) .
\end{aligned}
$$


The expectation of terms in the curly bracket on the right-hand side of (2.76) with the measure $E$ equals zero. Therefore we get for $X \neq Y$,

$$
\left|E \mu_{\Lambda, \Phi\left(t, s_{X}=0\right)} \psi_{X} \mu_{\Lambda, \Phi\left(t, s_{Y}=0\right)} \psi_{Y}\right| \leqq E\left(\sup _{\sigma} \psi_{X}\right)^{2} \cdot E\left(\sup _{\sigma} \psi_{Y}\right)^{2} .
$$

Hence

$$
|B(2.74)| \leqq\|\psi\|_{w}^{2} .
$$

This together with (2.75) and (2.78), by use one of Schwartz inequality yields

$$
E|A(2.68)| \leqq\left(1+\frac{1}{|\Lambda|^{1 / 2}}\right)\|\psi\|_{w}^{2} .
$$

Combining (2.73) and together with (2.67) (for $\phi^{\prime}=0$ ) we get

$$
E\left|p_{\Lambda}(\Phi)\right| \leqq\|\phi\|_{u}+2\|\psi\|_{w}^{2}+\frac{1}{|\Lambda|^{1 / 2}}\left(\|\psi\|^{2}+\|\psi\|_{w}\right) .
$$

This ends the proof of (2.34a) and so of Lemma 2.3.

Let us now discuss the a.s. convergence of the sequence of finite volume pressure functionals. For this purpose we will use the inequality (2.25) with $\Lambda_{k} \in \mathscr{F}_{0}$ and $N \equiv N_{k}$ chosen so that $N_{k} \rightarrow_{k \rightarrow \infty} \infty$,

$$
n_{k} \equiv n\left(\left(\Lambda_{k}^{-}\right)_{N_{k}}\right) \rightarrow_{k \rightarrow \infty} \infty \quad \text { and } \quad||\left(\Lambda_{k}^{-}\right)_{N_{k}}|/| \Lambda_{k}|-1| \rightarrow_{k \rightarrow \infty} 0 .
$$

Then we have (2.27), (2.28) and the $E$-a.s. convergence in (2.29) as $k \rightarrow \infty$. Since we know also that $\left\{E p_{A_{k}}\right\}_{k \in N}$ converges, so using (2.25), to conclude the a.s. convergence of the sequence $\left\{p_{A_{k}}\right\}_{k \in \mathbb{N}}$ it is sufficient to show that

$$
A_{k} \equiv\left|p_{A_{k}}-\frac{\left|\left(\Lambda_{k}^{-}\right)_{N_{k}}\right|}{|\Lambda|} \frac{1}{n_{k}} \sum_{l=1, \ldots, n_{k}} p_{\Delta_{N_{k}}^{(l)}}\right|
$$

converges $E$-a.s. to zero as $k \rightarrow \infty$.

We need to show that for any $\varepsilon>0$,

$$
\lim _{m \rightarrow \infty} E\left(\sup _{k \geqq m} A_{k}>\varepsilon\right)=0 .
$$

We will give here a simple proof of this fact, based on estimates of Lemma 2.2, at the cost of imposing some growth condition in $k$ of the van Hove sequence $\left\{\Lambda_{k}\right\}_{k \in \mathbb{N}}$. First of all let us note that

$$
E\left(\sup _{k \geqq m} A_{k}>\varepsilon\right) \leqq \frac{1}{\varepsilon} \sum_{k \geqq m} E\left(A_{k}\right),
$$

and by (2.12), (2.16) and Lemma 2.2 we have

$$
E\left(A_{k}\right) \leqq A(2.17)_{k}+A(2.16)_{k},
$$

where $A(2.17)_{k}$, respectively $A(2.16)_{k}$ denote the corresponding expressions from the (2.17) respectively the right-hand side of (2.18) computed with $\Lambda=\Lambda_{k}$ and $N=N_{k}$.

By imposing a growth condition in $k$ on the van Hove sequence $\left\{\Lambda_{k}\right\}_{k \in \mathbb{N}}$ we see that we can choose the corresponding function $L\left(N_{k}\right)$ from Lemma 2.2 so that the sequences $\frac{\left|\Lambda_{k} \backslash\left(\Lambda_{k}^{-}\right)_{N_{k}}\right|}{\left|\Lambda_{k}\right|}, \frac{L\left(N_{k}\right)}{N_{k}}$ and $\varepsilon_{\phi}\left(L\left(N_{k}\right)\right)$ (given by $\left.(2.42)\right)$ and $\tilde{\varepsilon}_{\psi}\left(L\left(N_{k}\right)\right)$ (given by 
(2.52)) are summable. This together with (2.83)-(2.84) and (2.82) show the $E$-a.s. convergence of the sequence $A_{k}$ given in (2.81). This ends the proof of $E$-a.s. convergence of the sequence of finite volume pressure functionals $\left\{p_{\Lambda_{k}}\right\}_{k \in \mathbb{N}}$.

Let us note that by our construction the van Hove sequence $\left\{\Lambda_{k}\right\}_{k \in \mathbb{N}}$ can be chosen in such the way that the $E$-a.s. convergence holds for an uncountable set of interactions $\Phi$ described only by the behavior of the tails $\varepsilon_{\phi}$ and $\tilde{\varepsilon}_{\psi}$ of the norm of $\Phi$. In particular restricting ourselves to a closed subspace of finite particle interactions, we see that one choice works for all the subspace.

Let us finish this section by a remark, that as follows from the above proofs, no essential changes are necessary to include the case when the variables $\left\{\phi_{X}\right\}_{X \in \mathscr{F}}$ defining an interaction $\phi \in \mathbb{B}^{u}(E)$ are mutually dependent.

\section{The Pressure Functional for Replica Systems}

In the analysis of a disordered system an important role is played by the pressure functional for replica systems. This is because the order parameter introduced by Edwards and Anderson [15] to indicate spin glass phase transition, can be formally defined as a derivative of such a functional. Therefore we include a theorem concerning existence and properties of the thermodynamic limit for the pressure of a replica system.

We will consider a lattice $\tilde{\Gamma} \equiv \boldsymbol{\Gamma} \times \mathbb{Z}$, that is, we take copies $\boldsymbol{\Gamma}_{k}$ of the lattice $\boldsymbol{\Gamma}$, indexed by integers $k \in \mathbb{Z}$. We use $\mathscr{F}$ to denote the family of finite sets in $\tilde{\boldsymbol{\Gamma}}$. A configuration space is now $(\widetilde{\Omega}, \widetilde{\Sigma}) \equiv(\Omega, \Sigma)^{\mathbb{Z}}=(S, S)^{\tilde{\mathbb{Z}}}$ and the corresponding coordinate functions (the spins) are denoted by $\sigma_{m}, \underline{m} \equiv(i, k) ; i \in \boldsymbol{\Gamma}, k \in \mathbb{Z}$. Let $\tilde{\mu}_{0} \equiv \mu_{0}^{\otimes \mathbb{Z}}$ be a free measure on $(\widetilde{\Omega}, \tilde{\Sigma})$. For $\Phi \in \mathbb{B}_{I}(E)$ we define an interaction $\widetilde{\Phi}$ on $(\widetilde{\Omega}, \widetilde{\Sigma}) \times(\mathbf{J}, \mathscr{J})$ as follows: If $\widetilde{X} \in \widetilde{\mathscr{F}}, \widetilde{X} \in \Gamma_{k}, k \in \mathbb{Z}$, i.e. $\widetilde{X}=\{\underline{m} \in \tilde{\Gamma}: \underline{m} \equiv(i, k), i \in X\}$ for some $X \in \mathscr{F}$, then we set

$$
\widetilde{\Phi}_{\tilde{X}}(J, \sigma) \equiv \Phi_{X}\left(J, \sigma_{(\cdot, k)}\right),
$$

and $\widetilde{\Phi}_{\tilde{X}} \equiv 0$ for all other $X \in \mathscr{F}$. In this way we get a translation invariant interaction. We would like to couple the hyperplanes $\boldsymbol{\Gamma}_{k} \equiv\{\underline{m} \equiv(i, k): i \in \boldsymbol{\Gamma}\}, \mathrm{k} \in \mathbb{Z}$, by introducing a translation invariant interaction $\widetilde{V}$ given by

$$
\tilde{V}_{\tilde{X}}(\sigma) \equiv V_{A}\left(\sigma_{(\cdot, l)}\right) \ldots V_{A}\left(\sigma_{(\cdot, l+n-1)}\right)
$$

for $\tilde{X} \equiv A \times[l, \ldots, l+n-1], n \in \mathbb{N}$ and with a translation invariant bounded (nonrandom) interaction $V \equiv\left\{\delta_{X, A+i} V_{A+i}, i \in \boldsymbol{\Gamma}\right\}$ defined on $(\Omega, \Sigma)$; for other sets $\tilde{X}$, by definition $\widetilde{V}_{\tilde{X}} \equiv 0$. For $\Lambda \in \mathscr{F}$ we take a set

$$
\tilde{\Lambda} \equiv \Lambda \times \mathcal{O},
$$

where $\mathcal{O} \equiv[L, M] \subset \mathbb{Z}, L<M$, and define a hamiltonian function

$$
H_{\tilde{\Lambda}}(\tilde{\Phi}, \tilde{V})=\sum_{\tilde{X} \subset \tilde{\Lambda}} \tilde{\Phi}_{\tilde{X}}+\sum_{\tilde{X} \subset \tilde{\Lambda}} V_{\tilde{X}},
$$

and a finite volume pressure

$$
p_{\tilde{\Lambda}}(\tilde{\Phi}, \tilde{V}) \equiv \frac{1}{|\tilde{\Lambda}|} \ln \tilde{\mu}_{0} e^{-H_{\tilde{\Lambda}}(\tilde{\Phi}, \tilde{V})}
$$


Let us note the following equality

$$
\frac{d}{d h} p_{\tilde{\Lambda}}(\tilde{\Phi}, h \tilde{V})_{\mid h=0}=\frac{1}{|\Lambda|} \sum_{A+i \subset \Lambda}\left(\mu_{\Lambda} V_{A+i}\right)^{n}
$$

where $\mu_{\Lambda}(\cdot)$ is a probability measure on $(\Omega, \Sigma)$ defined by

$$
\mu_{\Lambda}(\cdot) \equiv \frac{\mu_{0}\left(e^{-H_{\Lambda}(\Phi)}\right)}{\mu_{0}\left(e^{-H_{\Lambda}(\Phi)}\right)} .
$$

In particular taking $n=2, V_{A+i} \equiv \sigma_{i}$, we get the Edwards-Anderson order parameter

$$
q_{E-A}=\frac{1}{|\Lambda|} \sum_{i \in \Lambda}\left(\mu_{\Lambda} \sigma_{i}\right)^{2}=\frac{d}{d h} p_{\tilde{\Lambda}}\left(\tilde{\Phi},\left\{h \sigma_{(i, k)} \sigma_{(i, k+1)}\right\}\right)_{\mid h=0} .
$$

In the replica picture the order parameter introduced above measures a remanescence after decoupling of hyperplanes.

To get physical information about possible phase transitions we shall first take a thermodynamic limit for the pressure functional and then to study its differentiability properties. Now we have a little different situation than considered in the preceding section, because the interaction in various hyperplanes is described by mutually dependent random variables. Nevertheless we have the following result.

Theorem 3.1. Let E be a translation invariant ergodic probability measure on $(\mathbf{J}, \mathscr{J})$. Let $\Phi \in \mathbb{B}_{I}(E)$ and let $\widetilde{\Phi}$ be the corresponding interaction for the replica system given on $\tilde{\boldsymbol{\Gamma}}$ by (3.1). Then for any interval $\mathcal{O} \subset \mathbb{Z}$ the limit

$$
p_{\mathfrak{O}}(\widetilde{\Phi}, \widetilde{V}) \equiv \lim _{\mathscr{F}_{0}} p_{\Lambda \times \mathfrak{d}}(\widetilde{\Phi}, \widetilde{V})
$$

exists and is independent of $J \in \mathbf{J}$, E-a.e. and the van Hove sequence $\mathscr{F}_{0}$. Moreover

$$
p(\widetilde{\Phi}, \widetilde{V}) \equiv \lim _{\mathcal{O} \uparrow \mathbb{Z}} p_{\mathcal{O}}(\widetilde{\Phi}, \widetilde{V})
$$

also exist and both functionals are convex and continuous.

Remark. i) Without any problems one can include more complicated random interactions in the " $\mathbb{Z}$ direction" stochastically independent of those in $\Gamma_{k}$ planes.

ii) For any Fixed $\mathcal{O} \subset \mathbb{Z}$ it is possible to show a similar continuity estimate as in Theorem 2.1 with the part of the right-hand side of (2.9) dependent on $\psi$ 's multiplied by a constant dependent on $|\mathcal{O}|$.

Proof. First let us note the following stability bound

$$
p_{\Lambda}(\Phi)-\|V\|^{n} \leqq p_{\tilde{\Lambda}}(\tilde{\Phi}, \tilde{V}) \leqq p_{\Lambda}(\Phi)+\|V\|^{n}
$$

with

$$
\|V\| \equiv \sup _{\sigma \in \Omega}\left|V_{A}(\sigma)\right|
$$

Therefore we shall expect a correct thermodynamic behaviour. Below, we show only $L_{1}(E)$ convergence of finite volume pressure functionals. The problem of a.s. convergence can be discussed in a similar manner as the corresponding one in Sect. 2.

Define $\Delta_{N}^{m} \equiv \Delta_{N}^{(l)} \times I_{N}^{(k)}$ for $\underline{m} \equiv(l, k)$, where $\left\{\Delta_{N}^{(l)}: l=1, \ldots, n\left(\Lambda_{N}^{-}\right)\right\}$are defined by $N^{\text {th }}$ covering of $\Lambda_{N}^{-}$and $\left\{I_{N}^{(k)}: k=1, \ldots, k\left(\mathcal{O}_{N}^{-}\right)\right\},\left|I_{N}^{(k)}\right| \equiv N$ is an $N^{\text {th }}$ covering of the 
interval $\mathscr{O}_{N}^{-}$by disjoint intervals. Then we have

$$
p_{\tilde{\Lambda}}=\frac{\left|\mathcal{O}_{N}^{-}\right|}{|\mathcal{O}|} \frac{1}{k\left(\mathcal{O}_{N}^{-}\right)} \sum_{k=1, \ldots, k\left(\mathcal{O}_{\bar{N})}\right.} p_{\Lambda \times I_{N}^{(k)}}+\int_{0}^{1} d t \frac{1}{|\widetilde{\Lambda}|} \mu_{\tilde{\Lambda}, \tilde{\Phi}, \tilde{V}(t)}\left(H_{\tilde{\Lambda}}\left(\frac{d}{d t} \tilde{V}(t)\right)\right)
$$

when

$$
\widetilde{V}(t)_{Y} \equiv\left\{\begin{array}{l}
\widetilde{V}_{Y} \text { if } Y \subset A \times I_{N}^{(k)} \text { for some } k \\
t \widetilde{V}_{Y} \text { otherwise }
\end{array}\right.
$$

The second term on the right-hand side of (3.13) satisfies the estimate

$$
\int_{0}^{1} d t \frac{1}{|\widetilde{\Lambda}|} \mu_{\tilde{\Lambda}, \tilde{\Phi}, \tilde{V}(t)}\left(H_{\tilde{\Lambda}}\left(\frac{d}{d t} \widetilde{V}(t)\right)\right) \mid \leqq 2\left[\frac{n}{N}+\frac{\left|I_{N}^{(0)}\right|}{|\mathcal{O}|}\right]\|V\|^{n} .
$$

Now note that by analogous arguments as in the proof of Theorem 2.1 one can show that for any $I_{N}^{(k)}$ the limit

$$
\begin{aligned}
p_{I_{N}^{(k)}}(\tilde{\Phi}, \tilde{V}) & \equiv \lim _{\mathscr{F}_{0}} p_{A \times I_{N}^{(k)}}(\tilde{\Phi}, \tilde{V}) \\
& =\lim _{\mathscr{F}_{0}} E p_{\Lambda \times I_{N}^{(k)}}(\tilde{\Phi}, \widetilde{V})
\end{aligned}
$$

exists and is independent of the van Hove sequence $\mathscr{F}_{0}$. The corresponding proof requires only a modification in the choice of the interpolating potential $\tilde{\psi}_{\tilde{X}}\left(t, s_{\tilde{X}}\right)$ as follows:

$$
\tilde{\psi}_{\tilde{X}}\left(t, s_{\tilde{X}}\right)= \begin{cases}\psi_{Y}(t) & \text { if } \tilde{X} \neq X \times\{k\}, Y \neq X \\ s_{\tilde{X}} \psi_{X}(t) & \text { otherwise }\end{cases}
$$

$\left(\psi(t)\right.$ defined as previously) and similarly for $\tilde{\psi}_{\tilde{X}}\left(t, s_{\tilde{X}}, s_{\tilde{Y}}\right)$. Using (3.13), (3.15), and (3.11) and translation invariance of the interactions and the measure $E$ we get, uniformly in $\Lambda \in \mathscr{F}_{0}$, the bound

$$
E\left|p_{\Lambda \times \mathcal{O}}(\widetilde{\Phi}, \tilde{V})-p_{\Lambda \times \mathcal{O}}(\widetilde{\Phi}, \tilde{V})\right| \leqq\left|\frac{\left|\mathcal{O}_{N}^{-}\right|}{\left|\mathcal{O}_{N}\right|}-\frac{\left|\mathcal{O}_{N}^{\prime-}\right|}{\left|\mathcal{O}_{N}^{\prime}\right|}\right| E p_{A \times I_{N}^{(0)}}\left\{4 \frac{n}{N}+\frac{N}{|\mathcal{O}|}+\frac{N}{\left|\mathcal{O}^{\prime}\right|}\right\}\|V\|^{n} .
$$

This implies that the sequence $\left\{\lim _{\Lambda \in \mathscr{F}_{0}} p_{\Lambda \times \mathscr{O}}\right\}$ is Cauchy and finishes the proof of existence of the thermodynamic limit (and nonrandomness) of the pressure (3.10). Since the convergence is locally uniform in the interaction, using the continuity of $p_{\mathcal{O}} \equiv \lim _{\mathscr{F}_{0}} p_{\Lambda \times \mathcal{O}}$ (which can be proven analogously as in Lemma 2.3) we get continuity of $p(\widetilde{\Phi}, \widetilde{V})$. For this case we have no analog of the continuity estimate (2.9). It is possible to have it for fixed $\psi$ interaction, i.e. with respect to $\phi$ and $V$.) Convexity follows by standard arguments based on Hölder's inequality for the measure $\tilde{\mu}_{0}$. This ends the proof of Theorem 3.1.

Remark. Once we have a convex continuous functional $p(\widetilde{\Phi}, h \widetilde{V}), h \in \mathbb{R}$ we know that it is differentiable with respect to $h$ everywhere except possibly countably many points. This is unfortunately not such useful information since for an application we are interested in this property at the point $\hat{h} \equiv 0$. 


\section{The Variational Principle}

In this section we formulate an analog of the classical variational principle (see $[54,38]$ ). By that we get an a'priori meaning of infinite equilibrium state of a disordered system. This is especially important in the case of long range interactions for which the use of a Gibbsian description is problematic. To be concrete we keep the setting of Sect. 2, although the same can be done for replica systems. In the classical formulation of the variational principle one uses the notion of translation invariant states. For random systems we shall consider families of probability measures on the space of spin configurations satisfying the requirements of the following definition.

Definition 4.1. A family $\tilde{\varrho} \equiv\left\{\varrho_{J}\right\}_{J \in \mathbf{J}}$ consisting of probability measures on $(\Omega, \Sigma)$ is called measurable iff for any function $F \in \mathbb{M}((\Omega, \Sigma) \times(\mathbf{J}, \mathscr{J}))$ the function

$$
\mathbf{J} \ni J \rightarrow \varrho(F)
$$

is measurable, and is called translation invariant iff it is measurable and for any $a \in \mathbf{T}$ the random variables $\varrho(F)$ and $\varrho\left(T_{a} F\right)$ defined on the probability space $(\mathbf{J}, \mathscr{J}, E)$ are identically distributed.

The important examples of translation invariant families can be obtained by considering the infinite volume limits

$$
\mu_{J} \equiv \lim _{\mathscr{F}_{0}} \mu_{\Lambda, J}
$$

with

$$
\mu_{\Lambda, J}(\cdot) \equiv \frac{\mu_{0}\left(e^{-H \Lambda(\Phi)}\right)}{\mu_{0}\left(e^{-H \Lambda(\Phi)}\right)},
$$

defined for some interaction $\Phi \in \mathbb{B}_{I}(E)$, and the limit on right-hand side (4.2) is understood in the topology given by the semimetrics

$$
d_{E, F}\left(\mu_{J}, \mu_{J}^{\prime}\right) \equiv E\left|\mu_{J}(F)-\mu_{J}^{\prime}(F)\right|,
$$

where $F \in \mathbb{M}((\Omega, \Sigma) \times(\mathbf{J}, \mathscr{J}))$. For concrete examples, see e.g. $[30,58]$ (see also other references mentioned therein). Another example of a translation invariant family of measures will be constructed in a course of proof of Theorem 4.8 below.

We would like to consider families $\tilde{\varrho} \equiv\left\{\varrho_{J}\right\}_{J \in J}$ whose elements are locally equivalent to the free measure, i.e. such that: For any $\Lambda \in \mathscr{F}$ there is a (nonnegative) density function $\varrho_{J}^{(\Lambda)} \in L_{1}\left(\mu_{0}\right)$ satisfying

$$
\varrho_{\left.J\right|_{\Sigma_{\Lambda}}}(\cdot)=\mu_{\left.0\right|_{\Sigma_{\Lambda}}}\left(\varrho_{J}^{(A)}\right) .
$$

This is sufficiently general to include the families given by (4.2).

If this condition is satisfied, we define an entropy $S_{A}\left(\varrho_{J}\right)$ of a measure $\varrho_{J}$ in a volume $\Lambda \in \mathscr{F}$ by

$$
S_{\Lambda}\left(\varrho_{J}\right) \equiv-\mu_{0}\left(\varrho_{J}^{(\Lambda)} \ln \varrho_{J}^{(\Lambda)}\right) .
$$

If $\varrho_{\left.J\right|_{\Sigma_{A}}} \perp \mu_{0_{\Sigma_{S}}}$ we set $S_{\Lambda}\left(\varrho_{J}\right) \equiv-\infty$, and for $\Lambda \equiv \emptyset$ by definition $S_{\emptyset}\left(\varrho_{J}\right) \equiv 0$. The entropy has the following properties:

Lemma 4.1. i) (negativity)

$$
S_{\Lambda}\left(\varrho_{J}\right) \leqq 0,
$$


ii) (decrease) If $\Lambda, \Lambda^{\prime} \in \mathscr{F}$ and $\Lambda \subset \Lambda^{\prime}$ then

$$
S_{\Lambda^{\prime}} \leqq S_{\Lambda},
$$

iii) (strong subadditivity) For any $\Lambda, \Lambda^{\prime} \in \mathscr{F}$

$$
S_{\Lambda \cup A^{\prime}}\left(\varrho_{J}\right)+S_{\Lambda \cap \Lambda^{\prime}\left(\varrho_{J}\right)} \leqq S_{\Lambda}\left(\varrho_{J}\right)+S_{\Lambda^{\prime}}\left(\varrho_{J}\right) .
$$

A standard proof can be found in $[54,38]$.

Define a finite volume entropy density $s_{\Lambda}\left(\varrho_{J}\right)$ by

$$
s_{\Lambda}\left(\varrho_{J}\right) \equiv \frac{1}{|\Lambda|} S_{\Lambda}\left(\varrho_{J}\right)
$$

Let us note that if $\mu_{0}$ is a product of uniform probability measures on $\{-1,+1\}$ then a simple application of logarithmic Sobolev inequality [35] for the measure $\mu_{0}$ shows that the absolute value of $s_{A}\left(\varrho_{J}\right)$ is bounded by one. (More generally, if $\mu_{0}$ is a translationally invariant product measure on a space $M^{\mathbf{\Gamma}}$.with $M$ a finite set, then $\left|s_{\Lambda}\left(\varrho_{J}\right)\right|$ is bounded by a constant.) We have

Proposition 4.2. Let $E$ be a translation invariant ergodic probability measure on $(\mathbf{J}, \mathscr{J})$ and let $\tilde{\varrho} \equiv\left\{\varrho_{J}\right\}_{J \in \mathbf{J}}$ be a translation invariant family of probability measures on $(\Omega, \Sigma)$. Then the thermodynamic limit

$$
s\left(\varrho_{J}\right) \equiv \lim _{\mathscr{F}_{0}} s_{\Lambda}\left(\varrho_{J}\right)
$$

exists and is independent of $J \in \mathbf{J}$, E-a.e. and the van Hove sequence $\mathscr{F}_{0}$.

Motivated by the above proposition we introduce the following notation:

$$
s(\varrho) \equiv \lim _{\mathscr{F}_{0}} s_{\Lambda}\left(\varrho_{J}\right)=\lim _{\mathscr{F}_{0}} E s_{\Lambda}\left(\varrho_{J}\right) .
$$

Proof. Let us first note that from the decrease property of entropy, we see that the proof is trivial if for some $\Lambda \in \mathscr{F}_{0}$ we have $\varrho_{J \mid \Sigma_{A}}$. Therefore we need only to consider the opposite case. Using the decrease property from Lemma 4.1, we have for any $\Lambda \in \mathscr{F}_{0}$,

$$
\frac{\left|\Lambda_{N}^{+}\right|}{|\Lambda|} s_{\Lambda_{N}^{+}}\left(\varrho_{J}\right) \leqq s_{\Lambda}\left(\varrho_{J}\right) \leqq \frac{\left|\Lambda_{N}^{-}\right|}{|\Lambda|} s_{\Lambda_{\bar{N}}}\left(\varrho_{J}\right) .
$$

Since $\mathscr{F}_{0}$ is van Hove, so for any $N \in \mathbb{N},\left|\Lambda_{N}^{ \pm}\right| /|\Lambda| \rightarrow 1$ as $\Lambda \uparrow \Gamma$ and it is sufficient to show that $\lim _{\mathscr{F}_{0}} s_{\Lambda_{N}^{ \pm}}\left(\varrho_{J}\right)$ exists, is nonrandom and independent of $\mathscr{F}_{0}$. By subadditivity of entropy, Lemma 4.1iii), we have

$$
s_{\Lambda_{N}^{ \pm}}\left(\varrho_{J}\right) \leqq \frac{1}{n\left(\Lambda_{N}^{ \pm}\right)} \sum_{l=1, \ldots, n\left(\Lambda_{N}^{ \pm}\right)} s_{\Delta_{N}}\left(\varrho_{T_{0} J}\right)
$$

Hence using an extension of the Kingman's subadditive ergodic theorem (see $[47,46,41])$ we conclude that

$$
s\left(\varrho_{J}\right) \equiv \lim _{\mathscr{F}_{0}} s_{\Lambda_{N}^{+}}\left(\varrho_{J}\right)=\lim _{\mathscr{F}_{0}} s_{\Lambda_{\bar{N}}}\left(\varrho_{J}\right) \geqq-\infty
$$

exists, is translation invariant and therefore by ergodicity of $E$ is constant $E$-a.e. Simple comparison arguments show that (4.16) is independent of $N$ chosen and consequently of the van Hove sequence $\mathscr{F}_{0}$. Afterwards we will need also the 
following proposition which can be shown similarly as Theorem II.23, p. 41 in [38].

\section{Proposition 4.3. The function}

$$
\tilde{\varrho} \equiv\left\{\varrho_{J}\right\}_{J \in \mathbf{J}} \mapsto s(\tilde{\varrho})
$$

is affine, i.e. for any two families $\tilde{\varrho}_{1} \equiv\left\{\varrho_{J}^{(1)}\right\}_{J \in \mathbf{J}}$ and $\tilde{\varrho}_{2} \equiv\left\{\varrho_{J}^{(2)}\right\}_{J \in \mathbf{J}}$ and any $t \in[0,1]$,

$$
s\left(t \varrho_{J}^{(1)}+(1-t) \varrho_{J}^{(2)}\right)=t s\left(\varrho_{J}^{(1)}\right)+(1-t) s\left(\varrho_{J}^{(2)}\right) .
$$

It is also upper semicontinuous (with respect to the topology defined by the metrics (4.4)).

The last quantity necessary to formulate a variational principle is the energy density. For $\tilde{\varrho} \equiv\left\{\varrho_{J}\right\}_{\boldsymbol{J} \in \mathbf{J}}, \Phi \in \mathbb{B}_{I}(E)$ and a finite set $\Lambda \in \mathscr{F}$, let us define it by

$$
e_{\Lambda}\left(\varrho_{J}, \Phi\right) \equiv \frac{1}{|\Lambda|} \varrho_{J}\left(H_{\Lambda}(\phi(J, \cdot))\right) \text {. }
$$

We can write it in the form

$$
e_{\Lambda}\left(\varrho_{J}, \Phi\right)=\frac{1}{|\Lambda|} \sum_{i \in \Lambda} \varrho_{J}\left(\sum_{\substack{X \in \mathscr{F} \\ i \in X \subset A}} \frac{1}{|X|} \Phi_{X}(J, \cdot)\right) .
$$

Using this, translation invariance of the interaction $\Phi \in \mathbb{B}_{I}(E)$ and the family $\tilde{\varrho}$ $\equiv\left\{\varrho_{J}\right\}_{J \in \mathbf{J}}$ together with translation invariance and ergodicity of $E$, one may wish to take formally the infinite volume limit to get a quantity

$$
e(\tilde{\varrho}, \Phi) \equiv E \varrho_{J}\left(\sum_{\substack{X \in \mathscr{F} \\ 0 \in X}} \frac{1}{|X|} \Phi_{X}(J, \cdot)\right) .
$$

As one can see this is totally correct if $\Phi \in \mathbb{B}_{I}^{u}(E)$. However, if an interaction has a nonzero component from the space $\mathbb{B}_{I}^{w}(E)$, then it is hopeless to have the righthand side of (4.20) finite in general. Therefore we would like to restrict ourselves from now on to the translation invariant families $\tilde{\varrho} \equiv\left\{\varrho_{J}\right\}_{J \in \mathbf{J}}$ for which the functional

$$
\mathbb{B}_{I}(E) \ni \Phi \mapsto E \varrho_{J}\left(\sum_{\substack{X \in \mathscr{F} \\ 0 \in X}} \frac{1}{|X|} \Phi_{X}(J, \cdot)\right)
$$

is continuous. The space of such $\varrho$ is denoted by $\mathbb{B}_{I}^{*}(E)$. It is useful to understand how this space is big. To see that we prove the following

Proposition 4.4. Assume that the translation invariant family $\tilde{\mu} \equiv\left\{\mu_{J}\right\}_{J \in \mathbf{J}}$ is given by

$$
\mu_{J} \equiv \lim _{\mathscr{F}_{0}} \mu_{\Lambda, J},
$$

where $\mu_{\Lambda, J}$ are the finite volume measure defined by (4.3) with a potential $\Phi \in \mathbb{B}_{I}(E)$. Then the family $\tilde{\mu} \equiv\left\{\mu_{J}\right\}_{J \in \mathbf{J}}$ belongs to $B_{I}^{*}(E)$.

Proof. Let $\Phi^{\prime} \in \mathbb{B}_{I}(E), \Phi^{\prime} \equiv \phi^{\prime}+\psi^{\prime}$ and for any $X \in \mathscr{F}, s_{X} \in[0,1]$ an interpolation potential $\Phi^{\prime}\left(s_{X}\right)$ is given by

$$
\Phi^{\prime}\left(s_{X}\right)_{Y} \equiv\left\{\begin{array}{lll}
\Phi_{Y}^{\prime} & \text { if } & Y \neq X \\
s_{X} \Phi_{X}^{\prime} & \text { if } & Y=X
\end{array} .\right.
$$


It is clear that if the limit (4.22) exists, then for any fixed $X \in \mathscr{F}$, the limit of the sequence $\left\{\mu_{\Lambda, J, s_{X}}\right\}_{\Lambda \in \mathscr{F}_{0}}$ whose elements are defined as in (4.3) but with a potential $\Phi^{\prime}\left(s_{X}\right)$, also exists. Moreover the expectations for the corresponding measure

$$
\mu_{J, s_{X}} \equiv \lim _{\mathscr{F}_{0}} \mu_{\Lambda, J, s_{X}}
$$

are differentiable with respect to $s_{X} \in[0,1]$.

Let us now consider the functional

$$
\mathbb{B}_{I}(E) \ni \Phi \mapsto E \mu_{J}\left(\sum_{\substack{X \subset \mathscr{F} \\ 0 \in X}} \frac{1}{|X|} \Phi_{X}(J, \cdot)\right),
$$

where $\Phi \equiv \phi+\psi, \phi \in \mathbb{B}_{I}^{u}(E)$ and $\psi \in \mathbb{B}_{I}^{w}(E)$. The continuity with respect to the $\phi$ part of the interaction is standard (and is in fact independent of $\mu_{J}$ ). To see the continuity with respect to $\psi$ we write

$$
\mu_{J} \psi_{X}=\mu_{J, s_{X}=0} \psi_{X}+\int_{0}^{1} d s_{X} \mu_{J, s_{X}}\left(\psi_{X}^{\prime}, \psi_{X}\right)
$$

[where $\psi^{\prime}$ corresponds to the interaction $\Phi^{\prime}$ used to define $\mu_{J, s_{X}}$ in (4.24)].

Using this and the mean zero condition for the $\psi$ 's interactions we see that

$$
E\left(\mu_{J} \sum_{\substack{X \in \mathscr{F} \\ 0 \in X}} \frac{1}{|X|} \psi_{X}\right)=E \sum_{\substack{X \in \mathscr{F} \\ 0 \in X}} \frac{1}{|X|} \int_{0}^{1} d s_{X} \mu_{J, s_{X}}\left(\psi_{X}^{\prime}, \psi_{X}\right) .
$$

Now similar arguments as used in Sect. 2, based on definition of the $\psi^{\prime} s$ and Hölder's inequality, show that

$$
\left|E \mu_{J}\left(\sum_{\substack{X \in \mathscr{F} \\ 0 \in X}} \frac{1}{|X|} \psi_{X}\right)\right| \leqq\left\|\psi^{\prime}\right\|_{w} \cdot\|\psi\|_{w} .
$$

This ends the proof of Proposition 4.4.

We come back to the thermodynamic limit of the energy density. From the consideration (4.18)-(4.21) we conclude

Proposition 4.5. If $\tilde{\varrho} \in \mathbb{B}_{I}^{*}(E), \varrho \equiv\left\{\varrho_{J}\right\}_{J \in \mathbf{J}}$ for a translation invariant ergodic measure $E$ then

$$
\lim _{\mathscr{F}_{0}} e_{\Lambda}\left(\varrho_{J}, \Phi\right)=e(\varrho, \Phi),
$$

E-a.e. independently of the van Hove sequence $\mathscr{F}_{0}$.

The next step towards our variational principle is provided by the following fact Proposition 4.6. Let $\Lambda \in \mathscr{F}$. Then for any $\Phi \in \mathbb{B}_{I}(E)$ and any $\varrho \in \mathbb{B}_{I}^{*}(E)$,

$$
s_{\Lambda}\left(\varrho_{J}\right)-e_{\Lambda}\left(\varrho_{J}, \Phi(J)\right) \leqq p_{\Lambda}(\Phi(J, \cdot)) \text {, }
$$

and therefore

$$
s(\tilde{\varrho})-e(\tilde{\varrho}, \Phi) \leqq p(\Phi) .
$$

Proof. The inequality (4.30) follows from the definition of related quantities by application of Jensen's inequality (see $[54,38]$ ). The inequality (4.31) is a consequence of (4.30) and the existence of the thermodynamic limit for $p_{A}, s_{A}$, and $e_{\Lambda}$ proven respectively in Theorem 2.1, Proposition 4.2 and Proposition 4.5. 
As is known the equality sign in (4.30) holds iff $\tilde{\varrho} \equiv\left\{\varrho_{J}\right\}_{J \in \mathbf{J}}$ is a finite volume equilibrium family of states defined by

$$
\varrho_{J}:=\mu_{\Lambda, J}(\cdot) \equiv \frac{\mu_{0}\left(e^{-H_{\Lambda}(\Phi(J, \cdot))}\right)}{\mu_{0}\left(e^{-H_{\Lambda}(\Phi(J, \cdot)}\right)} .
$$

Therefore one may wish to use (4.31) and give an a'priori meaning of infinite volume equilibrium family of states in the following way.

Definition 4.7. An (infinite volume) equilibrium family of states for a random lattice system is by definition a family $\tilde{\varrho}_{\Phi} \in \mathbb{B}_{I}^{*}(E)$ satisfying

$$
s\left(\tilde{\varrho}_{\Phi}\right)-e\left(\tilde{\varrho}_{\Phi}, \Phi\right)=p(\Phi) .
$$

Our main result of this section is the theorem justifying this definition.

Theorem 4.8. Let $E$ be a translation invariant ergodic probability measure on $(\mathbf{J}, \mathscr{J})$ and let $\Phi \in \mathbb{B}_{I}(E)$. Then

$$
p(\Phi)=\sup _{\tilde{\varrho} \in \mathbb{B}^{*}(E)}\{s(\tilde{\varrho})-e(\tilde{\varrho}, \Phi)\} .
$$

Proof. Let $\Phi \in \mathbb{B}_{I}(E)$. We shall to show that for any $\varepsilon>0$ there is a family $\tilde{\varrho} \in \mathbb{B}_{I}^{*}(E)$ such that

$$
p(\Phi) \leqq s(\tilde{\varrho})-e(\tilde{\varrho}, \Phi)+\varepsilon .
$$

We construct these families $\varrho$ explicitly.

For $N \in \mathbb{N}$, let $\left\{\Delta_{N}\right\}_{N \in \mathbb{N}}$ be a covering of $\Gamma$ by disjoint $N$-cubes. We define first an infinite volume periodic family $\tilde{\mu}^{(P, N)} \equiv\left\{\mu_{J}^{(N)}\right\}_{J \in \mathbf{J}}$ by setting

$$
\mu_{J}^{(P, N)}\left|\Sigma_{\Delta}^{(L)}:=\mu_{\Delta_{N}^{(i)}, J}\right| \Sigma_{\Delta_{N}^{(i)}}
$$

and assuming the spins in different $N$-cubes to be independent.

To get a translation invariant family we average each measure $\mu_{J}^{(P, N)}$ over a cube $\Delta_{N}$, i.e. we define $\tilde{\mu}^{(N)} \equiv\left\{\mu_{J}^{(N)}\right\}_{J \in \mathbf{J}}$ by setting

$$
\mu_{J}^{(N)} \equiv \frac{1}{C_{N}} \Sigma T_{a} \mu_{J}^{(P, N)}
$$

where the sum runs over $a \in \mathbf{T}, a \equiv \sum_{i=1}^{d} n_{i} e_{i},\left|n_{i}\right| \leqq N$, and $C_{N}$ denotes the number of these translations. What is important for us, is the fact that $\tilde{\mu}^{(N)} \in \mathbb{B}_{I}^{*}(E)$. The conditions of Definition 4.1 are obviously satisfied, whereas the continuity of the corresponding functional (4.25) follows by similar arguments as in the proof of Proposition 4.4. Now we use Proposition 4.2 and Proposition 4.3 to get

$$
s\left(\tilde{\mu}^{(N)}\right)=E s_{\Delta_{N}}\left(\mu_{\Delta_{N}, J}\right) .
$$

We note also that by a choice of a sufficiently big $N \in \mathbb{N}$ the energy density $e\left(\tilde{\mu}^{(N)}, \Phi\right)$ and the pressure $p(\Phi)$ can be arbitrarily well approximated by $E \frac{1}{\left|\Delta_{N}\right|} \mu_{\Delta_{N}}\left(H_{\Delta_{N}}(\Phi)\right)$ and $E p_{\Delta_{N}}(\Phi)$ respectively. To see that one applies the same type of surface energy estimates as in the proof of Theorem 4.1. These together with the finite volume equality

$$
s_{\Delta_{N}}\left(\mu_{\Delta_{N}, J}\right)-e_{\Delta_{N}}\left(\mu_{\Delta_{N}, J}, \Phi(J, \cdot)\right)=p_{\Delta_{N}}(\Phi)
$$

give us (4.35) and so ends the proof of Theorem 4.8. 
With this we end the consideration of systems with interactions from the Banach space $\mathbb{B}_{I}(E)=\mathbb{B}_{I}^{u}(E)+\mathbb{B}_{I}^{w}(E)$.

\section{Long Range Random Site Interactions}

This section is devoted to a discussion of lattice systems with interactions $\Phi \in \overline{\mathbb{B}(E)}$, i.e. satisfying

$$
\|\Phi\| \equiv \sup _{\Lambda \in \mathscr{F}} \sup _{\sigma \in \Omega} \frac{1}{|\Lambda|}\left|\sum_{X \subset \Lambda} \Phi_{X}\right|<\infty, \quad E \text {-a.e. . }
$$

Let $\overline{\mathbb{B}_{I}(E)}$ denote the subspace of translation invariant elements from $\overline{\mathbb{B}(E)}$.

At the beginning we would like to show that there are reasonable interactions in $\overline{\mathbb{B}_{I}(E)}$, which do not belong to the space $\mathbb{B}_{I}(E)$ considered in Sects. 2-4. A class of such (long range two point) interactions has been considered in [32], where a complete description of corresponding lattice systems in the high temperature region was given. Here we present some possible generalizations of these interactions. Afterwards we discuss the thermodynamic limit for pressure. In the considered case our results are less satisfactory in general (some problems have been already encountered in [32]). As we will see in the present situation the replica systems are better. Let us introduce the occupation number random variables $n$ $\equiv\left\{n_{i}:(\mathbf{J}, \mathscr{J}) \rightarrow\{0,1\}\right\}_{i \in \Gamma}$, assumed to be independent and identically distributed. For $X \in \mathscr{F}$, let $\xi_{X}:(\Omega, \Sigma) \times(\mathbf{J}, \mathscr{J}) \rightarrow \mathbb{R}$ be a function dependent only on all spins $\left\{\sigma_{i}: i \in X\right\}$ and such that

$$
\xi_{X}(n, \sigma)=n_{X} \xi_{X}(n, \sigma),
$$

where

$$
n_{X} \equiv \prod_{i \in X} n_{i}
$$

Let a representation of the translation group $T$ be given by

$$
\left.\begin{array}{l}
T_{a} n_{i}:=n_{i-a} \\
T_{a} \sigma_{i}:=\sigma_{i-a}
\end{array}\right\}
$$

We assume that the functions (5.2) satisfy

$$
\xi_{X+L a}(n, \sigma)=\xi_{X}\left(T_{-L a} n, T_{-L a} \sigma\right)
$$

for all $a \in \mathbf{T}$ and some $L \in \mathbb{N}$.

Let $G: \mathbf{T} \rightarrow \mathbb{R}$,

$$
G_{a b} \equiv G(a-b):=\frac{1}{(2 \pi)^{d}} \int_{(-\pi, \pi)^{d}} d_{d} q e^{i q \cdot(a-b)} \hat{G}(q)
$$

with

$$
0 \leqq|\hat{G}(q)| \leqq\|\widehat{G}\|_{\infty}<\infty,
$$

and where for $a-b \equiv \sum_{i=1, \ldots, d}\left(a_{i}-b_{i}\right) e_{i}$ we set $q \cdot(a-b) \equiv \sum_{i=1, \ldots, d} q_{i}\left(a_{i}-b_{i}\right)$. To show that $\overline{\mathbb{B}_{I}(E)} \backslash \mathbb{B}_{I}(E)$ is nontrivial, we would like to distinguish the following examples of interactions.

Example 5.1. For $L \in \mathbb{N}$ we define an interaction $\Theta \equiv\left\{\Theta_{Y}\right\}_{Y \in \mathscr{F}}$ by

$$
\Theta_{Y}=\left\{\begin{array}{ll}
G_{a b} \xi_{X+L a} \xi_{X+L b} & \text { if } Y=(X+L a) \cup(X+L b) \\
0 & \text { otherwise }
\end{array}\right. \text {. }
$$

Using (5.4) and (5.6) we see that the interaction $\Theta$ is translation invariant. 
Note that in particular, if the spins $\sigma_{i} \in\{-1,+1\}$, then taking in the above example $\xi_{X} \equiv \sigma_{i}$ for $\{i\}=X$, we get Ising models. To include $S^{N}$ models one can generalize Example 5.1 as follows:

Example 5.2. Let $\left\{\xi_{X}^{(\alpha)}\right\}_{\alpha=1, \ldots, N}$ be the functions defined in (5.2)-(5.5). Let

$$
G_{a b}^{\alpha \alpha^{\prime}}:=\frac{1}{(2 \pi)^{d}} \int_{(-\pi, \pi)^{d}} d_{d} q e^{i q(a-b)} \widehat{G}^{\alpha \alpha^{\prime}}(q)
$$

with a matrix $\hat{G}(q) \equiv\left\{\hat{G}^{\alpha \alpha^{\prime}}(q)\right\}$ satisfying in the sense of quadratic forms

$$
-\lambda(q) \delta^{\alpha \alpha^{\prime}} \leqq \hat{G}^{\alpha \alpha^{\prime}}(q) \leqq \lambda(q) \delta^{\alpha \alpha^{\prime}}
$$

with some $0<\lambda<\infty$. We will set

$$
\|\hat{G}\| \equiv \sup _{q \in(-\pi, \pi)^{d}}(\inf \{\lambda \in(5.10)\}) .
$$

Now we define a translation invariant interaction by setting

$$
\Theta_{Y} \equiv \sum_{\alpha \alpha^{\prime}} G_{a b}^{\alpha \alpha^{\prime}} \xi_{X+L a}^{(\alpha)} \xi_{X+L b}^{\left(\alpha^{\prime}\right)}
$$

for $Y=(X+L a) \cup(X+L b)$ and zero otherwise.

We want also to indicate one more example in which we allow spins from different type of sets to interact.

Example 5.3. Let $\widehat{G}(q) \equiv\left\{\hat{G}^{\left(\alpha \alpha^{\prime}, X X^{\prime}\right)}(q)\right\}_{X, X^{\prime} \in \mathscr{F}}^{\alpha, \alpha^{\prime}=1, \ldots, N}$ be matrices satisfying in the sense of quadratic forms

$$
-\lambda_{X}(q) \delta^{\alpha \alpha^{\prime}} \delta_{X X^{\prime}} \leqq \hat{G}^{\left(\alpha \alpha^{\prime}, X X^{\prime}\right)}(q) \leqq \lambda_{X}(q) \delta^{\alpha \alpha^{\prime}} \delta_{X X^{\prime}}
$$

with some $0<\lambda_{X}(q)<\infty$ such that

$$
\|\hat{G}\| \equiv \sup _{q \in(-\pi, \pi)^{d}}\left(\inf \sum_{X \in \mathscr{F}}\left(\lambda_{X}(q) \in(5.13)\right)\right)<\infty .
$$

Let $G_{a b}^{\left(\alpha \alpha^{\prime}, X X^{\prime}\right)}$ be the Fourier transform of $\hat{G}^{\left(\alpha \alpha^{\prime}, X X^{\prime}\right)}(q)$. Assume

Then we define

$$
\sup _{\alpha, X}\left\|\xi_{X}^{(\alpha)}\right\|_{\infty}<\infty
$$

and zero otherwise.

$$
\Theta_{Y}:=\left\{\begin{array}{c}
\sum_{\alpha, \alpha^{\prime}} G_{a b}^{\left(\alpha \alpha^{\prime}, X X^{\prime}\right)} \xi_{X+L a}^{(\alpha)} \xi_{X^{\prime}+L b}^{\left(\alpha^{\prime}\right)} \\
\text { for } Y=(X+L a) \cup\left(X^{\prime}+L b\right)
\end{array}\right.
$$

To get a concrete realization of such an interaction one can for example choose

$$
\widehat{G}_{X X^{\prime}}^{\alpha \alpha^{\prime}}= \begin{cases}\hat{G}^{\alpha \alpha^{\prime}}(q) & \text { for }|X|<\tau \\ 0 & \text { otherwise }\end{cases}
$$

with some $\hat{G}^{\alpha \alpha^{\prime}}(q)$ from Example 5.2 and a number $\tau \in \mathbb{N}$.

We have the following proposition:

Proposition 5.4. Let $\phi \in \mathbb{B}_{I}^{\mathrm{cl}}(E)$. Then for all $\Theta$ interactions

$$
\Theta+\phi \in \overline{\mathbb{B}_{I}(E)} \text {. }
$$

Moreover there are interactions $\Theta$ belonging to $\overline{\mathbb{B}_{I}(E)} \backslash \mathbb{B}_{I}(E)$. 
Proof. First we show that $\Theta$ interactions satisfy (5.1).

We have, using the definition of $\Theta$

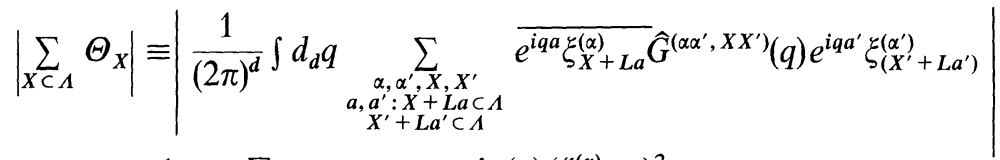

$$
\begin{aligned}
& \leqq \sum_{a, X: X+L a \subset A} \sup _{\alpha \in(-\pi, \pi)^{d}} \lambda_{X}(q)\left(\xi_{X}^{(\alpha)}+L a\right)^{2} .
\end{aligned}
$$

Hence

$$
\left|\sum_{X \subset A} \Theta_{X}\right| \leqq\|\hat{G}\| \sup _{\alpha, X}\left\|\xi_{X}^{(\alpha)}\right\|^{2} \cdot|\Lambda|,
$$

which proves (5.1) for interactions $\Theta$.

Since obviously also $\phi \in \mathbb{B}_{I}^{\text {cl }}(E)$ belongs to $\overline{\mathbb{B}_{I}(E)}$, we have (5.18).

(Remark. Note that restricting to a base $\mathscr{F}_{0} \subset \mathscr{F}$ we could get (5.1) for any $\Phi \in \overline{\left.\mathbb{B}_{I}^{u}(E) .\right)}$ To show that $\mathbb{B}_{I}(E) \backslash \mathbb{B}_{I}(E)$ is nonempty, it is sufficient to take $\Theta$ defined with a matrix $G$ satisfying

$$
\sum_{a \in T}\left|G_{a b}\right|=\infty,
$$

and any $\xi_{X}$ such that $E \xi_{X+L a} \xi_{X+L b} \neq 0$.

A simplest explicit example of a matrix $G$ satisfying (5.21) and (5.6)-(5.7) one gets by taking

$$
\widehat{G}(q) \equiv \prod_{i=1}^{d} \frac{2 \pi}{q_{0}^{(i)}} \chi\left(\left|q^{(i)}\right|<q_{0}^{(i)}\right) \cdot \mathbb{1}
$$

defined with some $0<\left|q_{0}^{(i)}\right|<\pi$. Then we have

$$
G_{a b}=\prod_{i=1}^{d} \frac{\sin q_{0}^{(i)}(a-b)}{q_{0}^{(i)}(a-b)} .
$$

Some other examples can be found in [32]. The interactions $\Theta$ given by a matrix $G$ from (5.23) are weakly decreasing with the distance $|a-b|$ and taking positive and negative values in an oscillatory way. Such interactions are thermodynamically stable and have all the important features of RKKY interactions, therefore can be used to describe models of alloys of ferromagnetic with nonferromagnetic materials (e.g. Fe with $\mathrm{Au}$ ).

To give a better idea how reach the class of functions $G$ satisfying (5.6)-(5.7) is, we would like to give also the following two examples:

Example 5.5. [60, p. 116]

For any $q_{0} \neq 0$ the sequence

$$
G(i-j)=\frac{\cos \left(q_{0}|i-j| \log |i-j|\right)}{|i-j|^{\alpha}},
$$

$i, j \in \mathbb{Z},|i-j| \geqq 1$, has a bounded (continuous) Fourier transform whenever

$$
\alpha>\alpha_{0} \equiv \frac{1}{2} .
$$

Another example showing that the value $\alpha_{0}=\frac{1}{2}$ is sharp, is the following 
Example 5.6. [60, p. 119]

For any $q_{0} \neq 0$ the sequence

$$
G(i-j)=\frac{\cos \left(q_{0}|i-j| \log |i-j|\right)}{|i-j|^{1 / 2}(\log |i-j|)^{\gamma}},
$$

$i, j \in \mathbb{Z},|i-j| \geqq 2$, has a bounded (continuous) Fourier transform $\hat{G}(q)$ whenever $\gamma>1$.

These examples show that the class of random side interactions considered in the present section is essentially the same as the class of random bond interaction introduced a long time ago by Edwards and Anderson. It would be interesting to understand better this connection and in particular to recover in the case of random side interactions the results corresponding to those mentioned in the introduction for random bond interactions.

To see that the introduced interactions are reasonable we would like to consider the thermodynamic limit for the pressure. Let us note that using the condition (5.1) one can always choose a countable base $\mathscr{F}_{0}$, in general dependent on the interaction $\Theta+\phi \in \mathbb{B}_{I}(E)$, such that

$$
p(\Theta+\phi) \equiv \lim _{\mathscr{F}_{0}} p_{\Lambda}(\Theta+\phi)
$$

exist and is nonrandom $E$-a.e. Using the condition (5.14) one can also show that this limit is the same for any two equivalent bases $\mathscr{F}_{0}=\left\{\Lambda_{n} \in \mathscr{F}\right\}_{n \in \mathbb{N}}$ and $\mathscr{F}_{0}^{\prime}$ $\equiv\left\{\Lambda_{n}^{\prime} \in \mathscr{F}\right\}_{n \in \mathbb{N}}$, where equivalence is defined by requirement that

$$
\lim _{n \rightarrow \infty} \frac{\left|\Lambda_{n} \div \Lambda_{n}^{\prime}\right|}{\left|\Lambda_{n}\right|}=0 .
$$

To get a well defined pressure functional we should show the independence of the thermodynamic limit for the pressure of the van Hove (or Fisher) countable base $\mathscr{F}_{0}$. However, as indicated in [32] there are some problems to get it, even if one considers some special examples of interactions. Roughly speaking they are connected to the fact that "a surface energy" (describing the interaction of $\Delta_{N}$ cube with its exterior) need not be small in absolute value (nevertheless the system remains stable due to "compensation in the mean" of interactions coming from different cubes). As noticed in [32] there is no problem if one considers interactions possessing a spin flip symmetry or interactions breaking this symmetry, but at high temperature.

To consider the first case, let us assume that there is a measurable bijection

$$
*:(\Omega, \Sigma) \rightarrow(\Omega, \Sigma): \Omega \ni \sigma \mapsto \sigma^{*} \in \Omega
$$

such that for any $\sigma \in \Omega$

$$
\left(\sigma^{*}\right)^{*}=\sigma .
$$

Any interaction $\phi$ respectively $\Theta$ satisfying

$$
\left.\begin{array}{c}
\phi_{X}\left(\sigma^{*}\right)= \pm \phi_{X}(\sigma) \\
\text { respectively } \Theta_{X}\left(\sigma^{*}\right)= \pm \Theta_{X}(\sigma)
\end{array}\right\}
$$

is called $*$-symmetric for $(+)$ sign in right-hand side (5.27) and is *-antisymmetric for $(-)$ sign, for all $X \in \mathscr{F}$. An interaction $\Theta$ is called $*$-symmetric of type I iff corresponding functions $\xi_{X}^{(\alpha)}$ are $*$-antisymmetric. 
We assume that the free measure $\mu_{0}$, used to define a finite volume pressure, is *-symmetric. This implies

$$
\mu_{0}(F)=0
$$

for any *-antisymmetric function $F$, i.e. satisfying $F\left(\sigma^{*}\right)=-F(\sigma)$.

We will need to consider the equivalence classes of van Hove sequences defined as follows: Two van Hove countable bases $\mathscr{F}_{0} \equiv\left\{\Lambda_{n} \in \mathscr{F}\right\}_{n \in \mathbb{N}}$ and $\mathscr{F}_{0}^{\prime}$ $\equiv\left\{\Lambda_{n}^{\prime} \in \mathscr{F}_{0}\right\}_{n \in \mathbb{N}}$ are called van Hove equivalent and we write $\mathscr{F}_{0} \sim \mathscr{F}_{0}^{\prime}$ iff one can choose a subsequence $\mathscr{F}_{0}^{\prime \prime} \equiv\left\{\Lambda_{k}^{\prime \prime} \in \mathscr{F}_{0}^{\prime}\right\}_{k \in \mathbb{N}}$ satisfying:

For any $k \in \mathbb{N}$ and any $\Lambda_{n} \in \mathscr{F}$ one can choose disjoint sets $\left\{T_{a i} \Lambda_{k}^{\prime \prime}\right.$ $\left.C \Lambda_{n}^{C}\right\}_{i=1, \ldots, k\left(\Lambda_{n}\right)}$ so that

$$
\lim _{k \rightarrow \infty} \lim _{n \rightarrow \infty} \frac{\left|\Lambda_{n} \backslash \bigcup_{i=1, \ldots, k\left(\Lambda_{n}\right)} T_{a i} \Lambda_{k}^{\prime \prime}\right|}{\left|\Lambda_{n}\right|}=0,
$$

and the same holds with $\mathscr{F}_{0}^{\prime}$ replaced by $\mathscr{F}_{0}$.

In particular any countable base consisting of sets of the form

$$
\Delta\left(N_{1}, \ldots, N_{\alpha}\right)=\left\{T_{\alpha} \Delta: a \equiv \sum_{i=1}^{d} n_{i} e_{i},\left|n_{i}\right| \leqq N_{i}\right\},
$$

where $N_{i} \in \mathbb{N}(i=1, \ldots, d)$ are van Hove equivalent. They are also equivalent to any van Hove base $\mathscr{F}_{0} \equiv\left\{\Lambda_{n} \in \mathscr{F}\right\}_{\Lambda \in \mathbb{R}}$ such that for any $n \in \mathbb{N}$ there exists a covering of $\Gamma$ by disjoint translations of $\Lambda_{n} \in \mathscr{F}_{0}$.

Let $\mathscr{F}_{\text {exp }} \equiv\left\{\Lambda_{n} \in \mathscr{F}\right\}_{n \in \mathbb{N}}$ denote a countable base, called exponential, consisting of sets $\Lambda_{n} \equiv \Delta_{N_{0}^{n}}$ for some $N_{0} \in \mathbb{N}, N_{0} \geqq 2$.

We would like to indicate the following result for $\Theta$ interactions

Proposition 5.7. Let $E$ be a translation invariant ergodic probability measure on $(\mathbf{J}, \mathscr{J})$. Let $\phi \in \mathbb{B}_{I}^{\mathrm{cl}}(E)$ be *-symmetric and let $\Theta *$-symmetric of type $I$. Then the thermodynamic limit for the pressure

$$
p(\Theta+\phi) \equiv \lim _{\mathscr{F}_{0}} p_{\Lambda}(\Theta+\phi)
$$

exist, is independent of $J \in \mathbf{J}$, E-a.e. and of the van Hove sequence $\mathscr{F}_{0}$ in any equivalence class. For any interaction $\phi \in \mathbb{B}_{I}^{\mathrm{cl}}(E)$ and $\Theta$, if the infinite volume pressure functional $p(\Theta+\phi)$ exists, it satisfies the following continuity property:

$$
\begin{aligned}
& \left|p(\Theta+\phi)-p\left(\Theta^{\prime}+\phi^{\prime}\right)\right| \leqq\left\|\hat{G}-\hat{G}^{\prime}\right\|\left(\sup _{\alpha, X} \max \left(\left\|\xi_{X}^{(\alpha)}\right\|_{\infty}+\left\|\xi_{X}\right\|_{\infty}\right)\right) \\
& +\max \left(\|\hat{G}\|,\left\|\hat{G}^{\prime}\right\|\right) \sup _{\alpha, X}\left\|\xi_{X}^{(\alpha)}-\xi_{X}^{\prime(\alpha)}\right\|_{\infty}+\left\|\phi-\phi^{\prime}\right\|_{\mathrm{cl}},
\end{aligned}
$$

where $\hat{G}, \xi_{X}^{(\alpha)}$ respectively $\hat{G}^{\prime}, \xi_{X}^{\prime(\alpha)}$ correspond to $\Theta$ respectively $\Theta^{\prime}$.

Remark. If one restricts oneself to $S^{N}$ models one may show the first part of Proposition 5.5 also for non-*-symmetric Gibbsian interactions $\phi \in \widetilde{\mathbb{B}}_{I}^{\text {cl }}(E)$ at high temperatures, by use of arguments from [59] (generalizing [32]). This allows us, which is important, to include a random external magnetic field.

Proof. We prove the existence and nonrandomness for the equivalence class of an exponential countable base $\mathscr{F}_{\exp } \equiv\left\{\Delta_{N_{0}^{n}}\right\}_{n \in \mathbb{N}^{*}}$. In other cases the proof is similar. Let $\mathscr{F}_{0}=\left\{\Lambda_{m} \in \mathscr{F}\right\}_{m \in \mathbb{N}}$ be a van Hove sequence. For $\Lambda \in \mathscr{F}_{0}$, let $\left\{\Delta_{N}^{(l)}\right\}_{l=1, \ldots, n\left(\Lambda_{\bar{N}}\right)}$ be a 
covering of $\Lambda_{N}^{-}$. Then we have

$$
\begin{aligned}
p_{\Lambda}(\Theta+\phi)= & \frac{1}{|\Lambda|} \ln \bigotimes_{l=1}^{n\left(\Lambda_{\bar{N}}\right)} \mu_{\Delta_{N}^{(l)}} \exp \left\{-\left(H_{\Lambda}-\sum_{l=1}^{n\left(\Lambda_{\bar{N}}\right)} H_{\Delta_{N}^{(l)}}\right)\right\} \\
& +\frac{\left|\Lambda_{N}^{-}\right|}{|\Lambda|} \frac{1}{n\left(\Lambda_{N}^{-}\right)} \sum_{l=1, \ldots, n\left(\Lambda_{\bar{N}}\right)} p_{\Delta_{N}^{(l)}}
\end{aligned}
$$

where $H_{\Lambda} \equiv H_{\Lambda}(\Theta+\phi)$ for any $\Lambda^{\prime} \in \mathscr{F}$ and the measures $\mu_{\Delta_{N}^{(l)}}$ are the finite volume measures defined for the interaction $\Theta+\phi$. Using Jensen's inequality we get

$$
\begin{aligned}
p_{\Lambda}(\Theta+\phi) \geqq & \frac{\left|\Lambda_{N}^{-}\right|}{|\Lambda|} \frac{1}{\mu\left(\Lambda_{N}^{-}\right)} \sum_{l=1, \ldots, n\left(\Lambda_{\bar{N}}\right)} p_{\Delta_{n}^{(l)}} \\
& -\frac{1}{|\Lambda|}\left(\bigotimes_{l=1}^{n\left(\Lambda_{\bar{N}}^{\bar{N}}\right)} \mu_{\Delta_{N}^{(l)}}\right)\left(H_{\Lambda}-\sum_{l=1}^{n\left(\Lambda_{\bar{N}}\right)} H_{\Delta_{N}^{(l)}}\right) .
\end{aligned}
$$

The second term on right-hand side (5.34) we write as follows:

$$
\begin{aligned}
\frac{1}{|\Lambda|} & \bigotimes_{l=1}^{n\left(\Lambda_{\bar{N}}\right)} \mu_{\Delta_{N}^{(l)}}\left[H_{\Lambda}-\sum_{l=1}^{n\left(\Lambda_{\bar{N}}\right)} H_{\Delta_{N}^{(l)}}\right] \\
= & \frac{1}{|\Lambda|} \bigotimes_{l=1}^{n\left(\Lambda_{\bar{N}}\right)} \mu_{\Delta_{N}^{(l)}}\left[H_{\Lambda}(\Theta)-\sum_{l=1}^{n\left(\Lambda_{\bar{N}}\right)} H_{\Delta_{N}^{(l)}}(\Theta)\right] \\
& +\frac{1}{|\Lambda|} \bigotimes_{l=1}^{n\left(\Lambda_{\bar{N}}\right)} \mu_{\Delta_{N}^{(l)}}\left(H_{\Lambda}(\phi)-\sum_{l=1}^{n\left(\Lambda_{\bar{N}}\right)} H_{\Delta_{N}^{(l)}}(\phi)\right) \equiv A(5.35)+B(5.35) .
\end{aligned}
$$

Since by our assumption the interactions $\Theta$ and $\phi$, and the free measure $\mu_{0}$ are *-symmetric, all the measures $\mu_{\Delta_{N}}$ are $*$-symmetric.

Now using the fact that $\Theta$ is $*$-symmetric of type I we get

$$
A(5.35)=\frac{1}{|\Lambda|} \bigotimes_{l=1}^{n\left(\Lambda_{\bar{N}}\right)} \mu_{\Delta_{N}^{(l)}} \sum^{\prime} \Theta_{(X+L a) \cup(X+L b)}
$$

with the summation $\sum^{\prime}$ going over the sets $X \in \mathscr{F}$ and $a, b \in T$ such that the conditions

$$
X+L a \cup X+L b \subset \Delta_{N}^{(l)}
$$

for some $l=1, \ldots, n\left(\Lambda_{N}^{-}\right)$or

$$
X+L a \subset \Delta_{N}^{(l)} \quad \text { and } \quad X+L b \subset \Delta_{N}^{\left(l^{\prime}\right)}
$$

for some $l, l^{\prime}=1, \ldots, n\left(\Lambda_{N}^{-}\right), l \neq l^{\prime}$, are not satisfied. From (5.36), using the definition of $\Theta$ interaction with $(5.13)-(5.16)$, it is not very complicated to get that

$$
\begin{aligned}
\lim _{\mathscr{F}_{0}} & \left|\frac{1}{|\Lambda|} \bigotimes_{l=1}^{n\left(\Lambda_{\bar{N}}\right)} \mu_{\Delta_{N}^{(l)}}\left(H_{\Lambda}(\Theta)-\sum_{l=1}^{n\left(\Lambda_{\bar{N}}\right)} H_{\Delta_{N}^{(l)}}(\Theta)\right)\right| \\
& \leqq \lim _{\mathscr{F}_{0}} \sup _{J, \sigma}\left|\Sigma^{\prime \prime} \Theta_{(X+L a) \cup(X+L b)}\right|=o(N),
\end{aligned}
$$

where $\sum^{\prime \prime}$ means the summation restricted to $X+L a$ and $X+L b$ intersecting some boundary $\partial \Delta_{N}^{(l)}$ resp. $\partial \Delta_{N}^{\left(l^{\prime}\right)}$, with some $o(N)>0, o(N) \rightarrow_{N \rightarrow \infty} 0$. (The conditions (5.11) and (5.15) play in the above the crucial role.) The second term on the right-hand side (5.35) has similar property by standard arguments.

Let now use these estimates together with (5.34) to show first that the limit of $\left\{E p_{\Lambda}\right\}_{\Lambda \in \mathscr{F}_{\exp }}$ exists. Integrating both sides with the translation invariant measure $E$ 
and using the translation invariance of the interaction and the free measure (used to define the finite volume pressure) we get in the limit as $\Lambda \nearrow \Gamma$ through a countable base $\mathscr{F}_{0}$ that

$$
\lim _{\mathscr{F}_{0}} \inf E p_{\Lambda} \geqq E p_{\Delta_{N}}-o(N) \text {. }
$$

Hence we get

$$
\lim _{\mathscr{F}_{0}} \inf E p_{\Lambda} \geqq \lim _{N \rightarrow \infty} \sup E p_{\Delta_{N}} .
$$

For an exponential base this implies that

$$
p(\Theta+\phi) \equiv \lim _{\mathscr{F}_{\text {exp }}} E p_{\Lambda}(\Theta+\phi)
$$

exists. Now we can use arguments based on (5.39) and (5.40) to show that in fact $p(\Theta+\phi)$ is the same for equivalent sequences $\mathscr{F}_{0} \sim \mathscr{F}_{\text {exp }}$.

Since $E$ is translation invariant and ergodic,

$$
\lim _{\mathscr{F}_{0}} \frac{1}{n\left(\Lambda_{N}^{-}\right)} \sum_{i=1, \ldots, n\left(\Lambda_{\bar{n}}\right)} p_{\Lambda_{N}^{(l)}}=E p_{\Delta_{N}}
$$

Therefore (5.34) also implies that

$$
\lim _{\mathscr{F}_{0}} \inf p_{\Lambda}(\Theta+\phi) \geqq E p_{\Delta_{N}}(\Theta+\phi)-o(N),
$$

and so

$$
\lim _{\mathscr{F}_{0}} \inf p_{\Lambda}(\Theta+\phi) \geqq \lim _{\mathscr{F}_{0}} E p_{\Lambda}(\Theta+\phi) \equiv p(\Theta+\phi) .
$$

This however allows us to conclude that

$$
p(\Theta+\phi)=\lim _{\mathscr{F}_{0}} p_{\Lambda}(\Theta+\phi)
$$

which ends the proof of the first part of Proposition 5.5. The continuity property follows from the estimate

$$
\begin{gathered}
\frac{1}{|\Lambda|}\left|H_{\Lambda}(\Theta+\phi)-H_{\Lambda}\left(\Theta^{\prime}+\phi^{\prime}\right)\right| \\
\leqq \\
\frac{1}{|\Lambda|}\left|H_{\Lambda}\left(\Theta-\Theta^{\prime}\right)\right|+\frac{1}{|\Lambda|}\left|H_{\Lambda}\left(\phi-\phi^{\prime}\right)\right|,
\end{gathered}
$$

and use the standard arguments to estimate the second part from the right-hand side of (5.46) and similar arguments as in the proof of Proposition 5.4 for the first part on the right-hand side of (5.46). This ends the proof of Proposition 5.7.

Remark. As observed in [32] one can show the existence and nonrandomness of the thermodynamic limit of the pressure even for some long range random site interactions violating (5.1) (given e.g. as in Example 1 but with $\hat{G}(q)=-\delta\left(q_{0}\right)$ for some $0 \leqq q<\pi$, and $\left.\left\{\xi_{X} \equiv \sigma_{\{i\}}\right\}\right)$. In this case one can however expect to have a pathological behaviour of the system as the interaction changes.

In investigation of spin glass phase transition an important role is played by replica systems. In this case we consider the same symmetric interactions inside each hyperplane $\Gamma_{k}$ and additionally couple the hyperplanes by the interaction of the form

$$
\tilde{V}_{X} \equiv h \cdot V_{A+L^{\prime} a}\left(\sigma_{(\cdot, k)}\right) V_{A+L^{\prime} a}\left(\sigma_{(\cdot, k+1)}\right)
$$


for $X \equiv A+L^{\prime} a$ with some $A \in \mathscr{F}, \Lambda^{\prime} \in \mathbb{N}$ and $a \in T$, and for other sets $X$ we put $\widetilde{V}_{X}$ $\equiv 0 ; h \in \mathbb{R}$. If we are interested in a spin glass phase transition we shall have to study the differentiability properties of the pressure functional at $h=0$. The interaction $\widetilde{V}$ plays the same role as the external magnetic field in the study of ferromagnetic phase transitions. We assume that the $V_{A}^{\prime \prime} s$ are $*$-antisymmetric. Then $\widetilde{V}$ is *-symmetric. Hence the replica system has again $*$-symmetry and we can adopt the proof of Proposition 5.7 to get the following result in whose formulation we use similar conventions as in Sect. 3.

Proposition 5.8. Let $E$ be a translation invariant ergodic probability measure on $(\mathbf{J}, \mathscr{J})$. Let $\widetilde{\Theta}+\tilde{\phi}$ be the interaction defined in $\Gamma_{k}, k \in \mathbb{Z}$ hyperplanes and corresponding to the translation invariant and *-symmetric interactions $\Theta+\phi$. Assume the interaction $\widetilde{V}$ defined in (5.47) is translation invariant and *-symmetric of type $I$. Then for any interval $\mathcal{O} \subset \mathbb{Z}$ the limit

$$
p_{\mathcal{O}}(\widetilde{\Theta}+\tilde{\phi}, \widetilde{V})=\lim _{\mathscr{F}_{0}} p_{\Lambda \times \mathcal{O}}(\widetilde{\Theta}+\tilde{\phi}, \tilde{V})
$$

exists, it is independent of $J \in \mathbf{J}, E$-a.e. and independent of the van Hove sequence $\mathscr{F}_{0}$ (inside an equivalence class of countable bases). Moreover

$$
p(\tilde{\Theta}+\tilde{\phi}, \widetilde{V}) \equiv \lim _{\mathcal{O} \uparrow \mathbb{Z}} p_{\mathcal{O}}(\widetilde{\Theta}+\tilde{\phi}, \tilde{V})
$$

also exists and both functionals are convex and continuous in the interactions.

Remark. Contrary to the situation in Sect. 3, now we can have a good continuity estimate for $p(\widetilde{\Theta}+\widetilde{\phi}, \widetilde{V})$ given by the right-hand side of $(5.32)$ plus the classical norm of the difference of the corresponding $\widetilde{V}^{\prime} s$ interactions.

Finally let us note that one may expect to have a very interesting behaviour of systems with $\Theta$ interactions in the low temperature region. As indicated in [59] there is a large subclass of $\Theta^{\prime} s$ [containing e.g. two point interactions with $G$ given by (5.23)] for which the spin system possess infinitely uncountably many nontranslation invariant ground states. Therefore one can expect to observe interesting phase transitions (including the breaking of translation symmetry for translation invariant nonrandom $\Theta$ interactions).

Acknowledgement. The author would like to thank A.C.D. van Enter for a number of valuable remarks and comments.

\section{References}

1. Aizenman, M., Lebowitz, J.L., Ruelle, D.: Some rigorous results on the SherringtonKirkpatrick spin glass model. Commun. Math. Phys. 112, 3-20 (1987), Addendum. Commun. Math. Phys. 116, 527 (1988)

2. Aizenman, M., Wehr, J.: Rounding effects of quenched randomness on first order phase transitions. Commun. Math. Phys. 130, 489-528 (1990)

3. Berretti, A.: Some properties of random Ising models. J. Stat. Phys. 38, 483-496 (1985)

4. Binder, K., Young, A.P.: Spin glasses: Experimental facts, theoretical concepts and open questions. Rev. Mod. Phys. 58, 801 (1986)

5. Bonato, C.A., Campanino, M.: Absence of symmetry breaking for systems of rotors with random interactions. J. Stat. Phys. 54, 81-88 (1989)

6. Bricmont, J., Kupiainen, A.: Phase transition in the $3 \mathrm{~d}$ random field Ising model. Commun. Math. Phys. 116, 539-572 (1988) 
7. Campanino, M., Olivieri, E.: One-dimensional random Ising systems with interaction decay $r^{-(1+\varepsilon)}$. A convergent cluster expansion. Commun. Math. Phys. 111, 555-577 (1987)

8. Campanino, M., Olivieri, E., van Enter, A.C.D.: One-dimensional spin glasses with potential decay $r^{-(1+\varepsilon)}$. Absence of phase transitions and cluster properties. Commun. Math. Phys. 108, 241-255 (1987)

9. Cassandro, M., Olivieri, E., Tirozzi, B.: Infinite differentiability for one-dimensional spin systems with long range random interaction. Commun. Math. Phys. 87, 229-252 (1982)

10. Chalker, J.T.: On the lower critical dimensionality of the Ising model in a random field. J. Phys. C 16, 6615-6622 (1983)

11. Chowdhury, D.: Spin glasses and other frustrated systems. Singapore: World Scientific 1986

12. Comets, F.: Large deviation estimates for a conditional probability distribution. Application to random interaction Gibbs measures. Probab. Theor. Rel. Fields 80, 407-432 (1989)

13. Dobrushin, R.L.: The description of a random field by means of conditional probabilities and conditions of its regularity. Theor. Prob. Appl. 13, 197-224 (1968). Prescribing a system of random variables by conditional distributions. Theor. Prob. Appl. 15, 458-486 (1970)

14. Dyson, F.J.: Existence of a phase transition in a one-dimensional Ising ferromagnet. Commun. Math. Phys. 12, 91-107 (1969)

15. Edwards, S.F., Anderson, P.W.: Theory of spin glasses. J. Phys. F: Metal. Phys. 5, 965-974 (1975)

16. van Enter, A.C.D.: Bounds on correlation decay for long-range vector spin-glasses. J. Stat. Phys. 11, 315 (1985)

17. van Enter, A.C.D.: One-dimensional spin glasses; uniqueness and cluster properties. J. Phys. A 21, 1781-1786 (1988)

18. van Enter, A.C.D., Fröhlich, J.: Absence of symmetry breaking for $N$-vector spin glass models in two dimensions. Commun. Math. Phys. 98, 425-432 (1985)

19. van Enter, A.C.D., Griffiths, R.B.: The order parameter in a spin glass. Commun. Math. Phys. 90, 319-327 (1983)

20. van Enter, A.C.D., van Hemmen, J.L.: The thermodynamic limit for long-range random systems. J. Stat. Phys. 32, 141-152 (1983)

21. van Enter, A.C.D., van Hemmen, J.L.: Absence of phase transitions in certain onedimensional long-range random systems. J. Stat. Phys. 39, 1-14 (1985)

22. Fölmer, H.: Phase transition and Martin boundary. In: Sém. Prob. Strasbourg IX. Lecture Notes in Mathematics, vol. 465. Berlin, Heidelberg, New York: Springer 1975

23. Figotin, A.L., Pastur, L.A.: Theor. Mat. Fiz. 35, 193 (1978)

24. Fisher, D.S., Fröhlich, J., Spencer, T.: The Ising model in a random magnetic field. J. Stat. Phys. 34, 863-870 (1984)

25. Fröhlich, J.: Mathematical aspects of the physics of disordered systems. pp. 725-894 in vol. II. Critical phenomena, random systems and gauge theories. Osterwalder, K. Stora, R. (eds.). Amsterdam: North-Holland 1986

26. Fröhlich, J., Imbrie, J.Z.: Improved perturbation expansion for disordered systems: beating Griffiths singularities. Commun. Math. Phys. 96, 145-180 (1984)

27. Fröhlich, J., Spencer, T.: The phase transition in one-dimensional Ising model with $1 / r^{2}$ interaction energy. Commun. Math. Phys. 84, 87-101 (1982)

28. Fröhlich, J., Simon, B., Spencer, T.: Infrared bounds, phase transitions and continuous symmetry breaking. Commun. Math. Phys. 50, 79-85 (1976)

29. Fröhlich, J., Zegarlinski, B.: The disordered phases of long-range Ising spin glasses. Europhys. Lett. 2, 53-60 (1986)

30. Fröhlich, J., Zegarlinski, B.: The high-temperature phase of long-range spin glasses. Commun. Math. Phys. 110, 121-155 (1987)

31. Fröhlich, J., Zegarlinski, B.: Some comments on the Sherrington-Kirkpatrick model of spin glasses. Commun. Math. Phys. 112, 553-566 (1987)

32. Fröhlich, J., Zegarlinski, B.: Spin glasses and other lattice systems with long-range interactions. Commun. Math. Phys. 120, 665-688 (1989)

33. Goulart-Rosa, S.: The thermodynamic limit of quenched free energy of magnetic systems with random interactions. J. Phys. A.: (Math. Gen.) 15, L51-54 (1982)

34. Griffiths, R.B.: Nonanalytic behaviour above the critical point in a random Ising ferromagnet. Phys. Rev. Lett. 23, 17-19 (1969)

35. Gross, L.: Logarithmic Sobolev inequalities. Am. J. Math. 97, 1061-1083 (1976) 
36. van Hemmen, J.L., Morgenstern, I. (eds.): Heidelberg Colloquium on Spin Glasses. Lecture Notes in Physics, vol. 192. Berlin, Heidelberg, New York: Springer 1983

37. Imbrie, J.Z.: The ground state of the three-dimensional random field Ising model. Commun. Math. Phys. 98, 145-176 (1985)

38. Israel, R.: Convexity in the theory of lattice gases. Princeton, NJ: Princeton Univ. Press 1979

39. Khanin, K.M.: Absence of phase transitions in one-dimensional long-range spin systems with random hamiltonian. Theor. Math. Phys. 43, 445-449 (1980)

40. Khanin, K.M., Sinai, Ya.G.: Existence of free energy for models with long-range random hamiltonians. J. Stat. Phys. 20, 573-584 (1979)

41. Krengel, U., Pyke, R.: Uniform and pointwise ergodic theorem for classes of averaging sets and multiparameter subadditive processes. Stochastic Processes Appl. 20, 573-584 (1979)

42. Krengel, U.: Ergodic theorems. Berlin, New York: Walter de Guyter 1985

43. Kunz, H., Pfister, C.-E.: First order phase transition in the plane rotator ferromagnetic model in two dimensions. Commun. Math. Phys. 46, 245-251 (1976)

44. Ledrappier, F.: Pressure and variational principle for random Ising model. Commun. Math. Phys. 56, 297-302 (1977)

45. Lanford III, O.E., Ruelle, D.: Observables at infinity and states with short range correlations in statistical mechanics. Commun. Math. Phys. 13, 194-215 (1969)

46. Nguyen, X.X., Zessin, H.: Ergodic theorems for spacial processes. Z. Wahrscheinlichkeitstheorie Verw. Geb. 48, 133-158 (1979)

47. Nguyen, X.X.: Ergodic theorems for subadditive spacial processes. Z. Wahrscheinlichkeitstheorie Verw. Geb. 48, 159-176 (1979)

48. Mezard, M., Parisi, G., Virasoro, M.A.: Spin glass theory and beyond. Singapore: World Scientific 1987

49. Olla, S.: Large deviations for Gibbs random fields. Probab. Theor. Rel. Fields 77, 343-357 (1988)

50. Park, Y.M.: Extension of Pirogov-Sinai theory of phase transitions to infinite range interactions. I. Cluster expansion. Commun. Math. Phys. 114, 187-216 (1988). II. Phase diagram. Commun. Math. Phys. 114, 219-241 (1988)

51. Picco, P.: On the absence of breakdown of symmetry for the plane rotator model with longrange interaction. J. Stat. Phys. 32, 627-648 (1983)

52. Picco, P.: Upper bound on the decay of correlations in the plane rotator model with longrange random interaction. J. Stat. Phys. 36, 489 (1984)

53. Preston, Ch.: Random fields. Lecture Notes in Mathematics, vol. 534. Berlin, Heidelberg, New York: Springer 1976

54. Ruelle, D.: Statistical mechanics: rigorous results. New York: W.A. Benjamin 1969

55. Sherrington, D., Kirkpatrick, S.: Solvable model of a spin-glass. Phys. Rev. Lett. 35, 1792-1796 (1975). Infinite-ranged models of spin-glasses. Phys. Rev. B 317, 4384-4403 (1978)

56. Steele, J.M.: Kingman's subadditive ergodic theorem. Ann. Inst. H. Poincaré 25, 93-98 (1989)

57. Vuillermot, P.A.: Thermodynamics of quenched random spin systems and application to the problem of phase transition in magnetic-(spin)-glasses. J. Phys. (Math. Gen.) A 10, 1319 (1977)

58. Zegarlinski, B.: Spin glasses with long-range interactions at high-temperature. J. Stat. Phys. 17, 911-930 (1987)

59. Zegarlinski, B.: On equivalence of spin and field pictures of lattice systems. J. Stat. Phys. 59, 1511-1530 (1990)

60. Zygmunt, A.: Trigonometrical series, Vol. 5, Monografje Matematyczne, Warszawa-Lwów 1935 
